\title{
Correlations of the characteristic polynomials in the Gaussian Unitary Ensemble or a singular Hankel determinant
}

\author{
I. V. Krasovsky \\ Department of Mathematical Sciences, Brunel University West London, \\ Uxbridge UB8 3PH, UK
}

\begin{abstract}
We obtain large $n$ asymptotics for products of powers of the absolute values of the characteristic polynomials in the Gaussian Unitary Ensemble of $n \times n$ matrices. Our results can also be interpreted as asymptotics of the determinant of a Hankel matrix whose symbol is supported on the real line and possesses power-like (Fisher-Hartwig) singularities.
\end{abstract}

\section{Introduction}

In the present paper we compute large $n$ asymptotics for the following averages of the characteristic polynomials over the Gaussian Unitary Ensemble (GUE) of $n \times n$ matrices $H$ :

$$
\left\langle\prod_{j=1}^{m}\left|\operatorname{det}\left(H-\mu_{j}\right)\right|^{2 \alpha_{j}}\right\rangle_{\mathrm{GUE}}=\frac{D_{n}\left(\alpha_{1}, \ldots, \alpha_{m}\right)}{D_{n}(0, \ldots, 0)}, \quad \Re \alpha_{j}>-\frac{1}{2}, \quad j=1, \ldots, m
$$

where

$D_{n}\left(\alpha_{1}, \ldots, \alpha_{m}\right)=\frac{1}{n !} \int_{-\infty}^{\infty} \cdots \int_{-\infty}^{\infty} \prod_{i<j}\left(x_{i}-x_{j}\right)^{2} \prod_{k=1}^{n} w\left(x_{k}\right) d x_{k}, \quad w(x)=\prod_{j=1}^{m}\left|x-\mu_{j}\right|^{2 \alpha_{j}} e^{-x^{2}}$,

and all $\mu_{j}$ are distinct. The condition $\Re \alpha_{j}>-\frac{1}{2}$ guarantees convergence.

It is easy to verify that

$$
D_{n}\left(\alpha_{1}, \ldots, \alpha_{m}\right)=\operatorname{det}\left(M_{i+j}\right)_{i, j=0}^{n-1}, \quad M_{k}=\int_{-\infty}^{\infty} x^{k} w(x) d x
$$

that is, $D_{n}\left(\alpha_{1}, \ldots, \alpha_{m}\right)$ is a Hankel determinant with symbol $w(x)$. 
The determinant $D_{n}(0, \ldots, 0)$ is an $n$-dimensional Selberg integral [29], it can be evaluated exactly, and its large- $n$ asymptotics readily can be written (cf. [36]):

$$
D_{n}(0, \ldots, 0)=(2 \pi)^{n / 2} 2^{-n^{2} / 2} \prod_{j=1}^{n-1} j !=(2 \pi)^{n}(n / 2)^{n^{2} / 2} n^{-1 / 12} e^{-(3 / 4) n^{2}+\zeta^{\prime}(-1)}(1+O(1 / n))
$$

where $\zeta^{\prime}(x)$ is the derivative of Riemann's zeta-function.

The average (1) is related to several interesting questions. We mention a conjectured relation of (1) for $m=1$ and such averages over other ensembles of random matrices to the mean values $\{1 / T\} \int_{0}^{T}|\zeta(1 / 2+i t)|^{2 \alpha} d t$ of the zeta-function on the critical line [23, 24, 7]. Also note that $D_{n}\left(\alpha_{1}, \ldots, \alpha_{m}\right)$ is a Hankel determinant with symbol on $\mathbb{R}$ and Fisher-Hartwig singularities at $x=\mu_{j}$. Although asymptotics of Toeplitz determinants with general FisherHartwig symbols have by now largely been determined (at least the leading terms thereof, see, e.g., [17]), the Hankel case is still open.

For connections of (1) to the one-dimensional gas of impenetrable bosons and other physical problems, see [17, 18, 19, 20] and below. (see also [3], 66, [20], [30], [31], 33] regarding subleading asymptotic terms, negative moments and other random matrix ensembles).

The asymptotics of averages (11) for $\alpha_{j}$ positive integers have been found by Brézin and Hikami [7], Forrester and Frankel [17], Garoni [21]. For such $\alpha_{j}$ 's, the expression (11) can be reduced to the Hermite polynomials and their derivatives at the points $\mu_{j}$. (In the framework of the present paper, this can be shown with the help of Christoffel's formula (e.g., [32], p.29), cf. [3]) It is not, however, the case for noninteger $\alpha_{j}$ 's. We prove

Theorem 1 Fix $\lambda_{j}$ and $\alpha_{j}$ such that $\lambda_{j} \in(-1,1), \lambda_{j} \neq \lambda_{k}$, for $j \neq k$, and $\Re \alpha_{j}>-1 / 2$, $j, k=1, \ldots, m$. Then, as $n \rightarrow \infty$,

$$
\begin{aligned}
& \left\langle\prod_{j=1}^{m}\left|\operatorname{det}\left(H-\lambda_{j} \sqrt{2 n}\right)\right|^{2 \alpha_{j}}\right\rangle_{\mathrm{GUE}}=\prod_{j=1}^{m}\left[C\left(\alpha_{j}\right)\left(1-\lambda_{j}^{2}\right)^{\alpha_{j}^{2} / 2}\left(\frac{n}{2}\right)^{\alpha_{j} n+\alpha_{j}^{2}} \exp \left\{\left(2 \lambda_{j}^{2}-1\right) \alpha_{j} n\right\}\right] \times \\
& \prod_{1 \leq i<j \leq m}\left(2\left|\lambda_{i}-\lambda_{j}\right|\right)^{-2 \alpha_{i} \alpha_{j}}\left[1+O\left(\frac{\ln n}{n}\right)\right],
\end{aligned}
$$

where

$$
C(\alpha)=\Gamma(\alpha+1 / 2)^{-2 \alpha} \exp \left(2 \int_{0}^{\alpha} \ln \Gamma(s+1 / 2) d s+\alpha^{2}\right)=2^{2 \alpha^{2}} \frac{G(\alpha+1)^{2}}{G(2 \alpha+1)},
$$

and $G(z)$ is Barnes' $G$-function. In (5), the values of the roots positive for real $\alpha_{j}$ (and their analytic extension for complex $\alpha_{j}$ ) are taken.

With increasing effort, one can compute an arbitrary number of terms in these asymptotics (and verify a heuristic calculation of them by Gangardt [20] for $m=1$ ).

The expression (5) without the error term was conjectured in [17], Conjecture 4, as an extension off integer values of $\alpha_{j}$. 
The asymptotics for the Hankel determinant $D_{n}\left(\alpha_{1}, \ldots, \alpha_{2}\right)$ are now just a combination of (1), (4), and (5). The corresponding asymptotics for a Toeplitz determinant with symbol (on the unit circle) having such type of singularities (multiplied by a rather general "regular" function) were found by Widom [37]. The multiplicative constant in the asymptotics involves the same combination of $G$ functions as here. Note that averages of type (1) for the Circular Unitary Ensemble are such singular Toeplitz determinants. The conjectured relation to Riemann's zeta-function averages was first formulated by Keating and Snaith [23] for that ensemble.

Let $m=1$. Since the density of the scaled eigenvalues in the GUE is given by $\psi(\lambda)=$ $(2 / \pi) \sqrt{1-\lambda^{2}},-1<\lambda<1$ (the Wigner semicircle law), we have that $D_{n}(\alpha)$ is proportional to $\psi^{\alpha^{2}}$. Similar dependence on zero density as well as presence of the factor $G(\alpha+1)^{2} / G(2 \alpha+$ 1 ) is conjectured for the large $T$ asymptotics of the zeta-function average mentioned above.

For $\alpha=1 / 2, m=1$, Theorem 1 gives an analogue for the GUE of the average of the absolute value of the characteristic polynomial found by Fyodorov for the Gaussian Orthogonal Ensemble [19] (in the Unitary Ensemble, his method would correspond to the case $\alpha=1)$.

The condition $\lambda \in(-1,1)$ means that $\lambda$ is inside the bulk of the scaled spectrum of $H$ (in other words, inside the support of the equilibrium measure for the GUE). The case $\lambda \in \mathbb{C} \backslash[-1,1]$ corresponds to a Hankel determinant with symbol "regular" on the support of the equilibrium measure, and the average (1) is then found as a particular case of a result by Johansson [22], although some nonessential assumptions in [22] need to be relaxed. (This result gives a Hankel analogue of the Szegö asymptotics for Toeplitz determinants with a regular symbol. The need to consider the equilibrium measure is an additional difficulty in comparison with the Toeplitz case.) For real $\lambda, \alpha, m=1$, we obtain from [22] as $n \rightarrow \infty$ :

$$
\begin{aligned}
& \left\langle|\operatorname{det}(H-\lambda \sqrt{2 n})|^{2 \alpha}\right\rangle_{\mathrm{GUE}}=(2 n)^{\alpha n}\left(\lambda^{2}-1\right)^{-\alpha^{2}}\left(\frac{|\lambda|+\sqrt{\lambda^{2}-1}}{2}\right)^{2 \alpha(n+\alpha)} \times \\
& \exp \left\{2 \alpha n\left(\lambda^{2}-|\lambda| \sqrt{\lambda^{2}-1}-\frac{1}{2}\right)\right\}[1+o(1)], \quad \lambda \in \mathbb{R} \backslash[-1,1], \quad \alpha>-1 / 2 .
\end{aligned}
$$

This (including the error terms) and the asymptotics for the border case of $\lambda$ close to \pm 1 could also be computed by our methods. It should also be possible to generalize Theorem 1 replacing $x^{2}$ in $w(\sqrt{2 n} x)$ by a more general function $V(x)$. Thus it should be possible to verify Conjecture 8 of Forrester and Frankel [17].

The one-body density matrix $\rho\left(\lambda_{1}, \lambda_{2}\right)$ for the ground state wave function of the onedimensional gas of impenetrable bosons equals [18], up to a simple factor, to $D_{n}(1 / 2,1 / 2)$ (i.e., the corresponding $w(x)=\left|x-\lambda_{1}\right|\left|x-\lambda_{2}\right| e^{-x^{2}}$ ), where $n$ represents the number of particles. The eigenvalues of the operator with kernel $\rho\left(\lambda_{1}, \lambda_{2}\right)$ are interpreted as occupation numbers of effective single-particle states of the Bose system. To prove the expected asymptotic $\sqrt{n}$-proportionality of the leading occupation numbers in this one-dimensional system (note that Bose-Einstein condensation, which is not expected in one dimension, would correspond to the $n$-proportionality), we need to analyze asymptotics of $D_{n}(1 / 2,1 / 2)$ for all 
values of $\lambda_{1}, \lambda_{2}$. Theorem 1 gives the desired asymptotics (conjectured in [18], eq.(105)) for $-1<\lambda_{1}<\lambda_{2}<1$, but we still need to analyze, in particular, cases of $\lambda_{1}, \lambda_{2}$ approaching each other and the points \pm 1 . This will be the subject of a subsequent publication.

We prove Theorem 1 using Riemann-Hilbert problem methods (see [8] for an introduction). Our approach is as follows. Consider the polynomials $p_{n}(x)=\varkappa_{n} x^{n}+\cdots$ orthonormal w.r.t. to the weight $w(x)=\prod_{j=1}^{m}\left|x-\lambda_{j} \sqrt{2 n}\right|^{2 \alpha_{j}} \exp \left(-x^{2}\right)$ :

$$
\int_{-\infty}^{\infty} p_{j}(x) p_{k}(x) w(x) d x=\delta_{j k}, \quad j, k=0,1, \ldots
$$

Then,

$$
D_{n}\left(\alpha_{1}, \ldots, \alpha_{m}\right)=\prod_{j=0}^{n-1} \varkappa_{j}^{-2},
$$

by a well-known formula [32, 8] (for real $\alpha_{j}$; the case of complex $\alpha_{j}$ will be explained below). The asymptotics of the polynomials $p_{n}(x)$ (for $\lambda=0$ and $m=1$ ) as $n \rightarrow \infty$ were recently analyzed by Kuijlaars and Vanlessen in [27] by a Riemann-Hilbert problem approach. Using [27], one can obtain any number of asymptotic terms for $p_{n}(x)$ and $\varkappa_{n}$.

Thus, knowing $\varkappa_{j}, j \rightarrow \infty$, we can shed light on the asymptotics of $D_{n}\left(\alpha_{1}, \ldots, \alpha_{m}\right)$. However, since the product of the first $\varkappa_{j}, j=1,2, \ldots$ remains unknown we are bound to lose at least the constant (in $n$ ) factor in such obtained asymptotics of $D_{n}$ (cf. [28]). This problem is avoided here by deriving an identity for $\left(d / d \alpha_{\nu}\right) \ln D_{n}\left(\alpha_{1}, \ldots, \alpha_{m}\right), \nu=1, \ldots, m$. (cf. Deift's formula [9] and differential identities of [11]), an idea which has proven useful in similar situations [15, 5, 25, 13, 14. It turns out that the particular structure of the weight $w(x)$ allows us to write the above logarithmic derivative only in terms of $p_{n}(z)$, $\int_{-\infty}^{\infty} p_{n}(x) w(x) d x /(x-z)$, and similar expressions with index $n-1$, which are precisely the quantities whose asymptotics (uniform for $\alpha$ in a bounded set) follow from the RiemannHilbert problem for $p_{n}(z)$. A fact that considerably simplifies calculations is that these quantities only need to be evaluated at a finite number of points $z$ ( $\mu_{j}$ and infinity); in this sense the identity we obtain is "local". (As discussed in Section 3, a "nonlocal" identity involving integrals with $p_{n}(x), p_{n-1}(x)$ exists for general weights). The identity is found in Section 3. Setting in it all $\alpha_{j}=0, \nu=1$, and integrating over $\alpha_{1}$ from zero to some $\alpha_{1}$, we obtain the asymptotics for $\ln \left(D_{n}\left(\alpha_{1}, 0, \ldots, 0\right) / D_{n}(0, \ldots, 0)\right)$. Now fixing in the identity $\alpha_{1}$, setting $\alpha_{2}=\cdots=\alpha_{m}=0, \nu=2$, and integrating over $\alpha_{2}$ from zero to some $\alpha_{2}$, we obtain $\ln \left(D_{n}\left(\alpha_{1}, \alpha_{2}, 0, \ldots, 0\right) / D_{n}\left(\alpha_{1}, 0, \ldots, 0\right)\right)$. Continuing this procedure, we prove the theorem by induction. Note that instead of zero, we could have used any positive integer $\alpha$ (where, as noted above, the asymptotics of $D_{n}$ are known) as a starting point for the integration.

\section{Riemann-Hilbert problem for $p_{n}(z)$}

Consider the following Riemann-Hilbert problem for a $2 \times 2$ matrix valued function $Y(z)$ and the weight $w(x)=\prod_{j=1}^{m}\left|x-\mu_{j}\right|^{2 \alpha_{j}} \exp \left(-x^{2}\right), \Re \alpha_{j}>-1 / 2$ : 
(a) $Y(z)$ is analytic for $z \in \mathbb{C} \backslash \mathbb{R}$.

(b) Let $x \in \mathbb{R} \backslash \cup_{j=1}^{m}\left\{\mu_{j}\right\}$. $Y$ has continuous boundary values $Y_{+}(x)$ as $z$ approaches $x$ from above, and $Y_{-}(x)$, from below. They are related by the jump condition

$$
Y_{+}(x)=Y_{-}(x)\left(\begin{array}{cc}
1 & w(x) \\
0 & 1
\end{array}\right), \quad x \in \mathbb{R} \backslash \cup_{j=1}^{m}\left\{\mu_{j}\right\} .
$$

(c) $Y(z)$ has the following asymptotic behavior at infinity:

$$
Y(z)=\left(I+O\left(\frac{1}{z}\right)\right)\left(\begin{array}{cc}
z^{n} & 0 \\
0 & z^{-n}
\end{array}\right), \quad \text { as } z \rightarrow \infty .
$$

(d) The matrix of $Y(z)$ is $O(1)$ for $\Re \alpha_{j} \geq 0$, and $\left(\begin{array}{cc}O(1) & O\left(\left|z-\mu_{j}\right|^{2 \alpha_{j}}\right) \\ O(1) & O\left(\left|z-\mu_{j}\right|^{2 \alpha_{j}}\right)\end{array}\right)$ for $\Re \alpha_{j}<0$ as $z \rightarrow \mu_{j}, j=1, \ldots, m, z \in \mathbb{C} \backslash \mathbb{R}$.

(Here and below $O(a)$ stands for $O(|a|)$.)

It is easy to verify that, provided the system of orthogonal polynomials $p_{k}(z)=\varkappa_{k} z^{k}+$ $\cdots, \varkappa_{k} \neq 0, k=0,1, \ldots$, satisfying (8) exists, this problem has a solution given by the function:

$$
Y(z)=\left(\begin{array}{cc}
\varkappa_{n}^{-1} p_{n}(z) & \varkappa_{n}^{-1} \int_{-\infty}^{\infty} \frac{p_{n}(\xi)}{\xi-z} \frac{w(\xi) d \xi}{2 \pi i} \\
-2 \pi i \varkappa_{n-1} p_{n-1}(z) & -\varkappa_{n-1} \int_{-\infty}^{\infty} \frac{p_{n-1}(\xi)}{\xi-z} w(\xi) d \xi
\end{array}\right) .
$$

For general weights this fact was noticed by Fokas, Its, and Kitaev [16] and, in conjunction with the steepest descent method of Deift and Zhou [10] for asymptotic analysis of matrix Riemann-Hilbert problems, has allowed in recent years to solve many previously unaccessible asymptotic problems for orthogonal polynomials (see [8] for an introduction and bibliography of earlier works, and [27, 34, 28, 26, 4, 2, 25, 13]).

Note, in particular, that $\operatorname{det} Y(z)=1$. (From the conditions on $Y(z)$ it follows that $\operatorname{det} Y(z)$ is analytic across the real axis, has all singularities removable, and tends to the identity as $z \rightarrow \infty$. It is then identically 1 by the Liouville theorem.) The solution is unique. Indeed, if there is another solution $\tilde{Y}(z)$, we easily obtain by the Liouville theorem that $Y(z) \widetilde{Y}(z)^{-1} \equiv 1$.

The case of the weight $w(x)=|x|^{2 \alpha} \exp \left(-x^{2}\right), \alpha>-1 / 2$, was considered in [27] (the argument is straightforward to generalize to our case). Namely, [27] gives us a ready procedure to calculate asymptotics of $Y(z)$ in this case (by applying a series of transformations to the problem (a)-(d)). By (12), these results are then interpreted as asymptotics of the polynomials $p_{n}(z)$ and their Cauchy transforms.

In the next section we shall derive an expression for $\left(d / d \alpha_{j}\right) \ln D_{n}\left(\alpha_{1}, \ldots, \alpha_{m}\right)$ in terms of the matrix elements of (12), and in the section after that compute the asymptotics of $Y(z)$. 
The existence of the system of orthogonal polynomials $p_{k}(z)=\varkappa_{k} z^{k}+\cdots$ satisfying (8) with nonzero leading coefficients $\varkappa_{k}$ for $\alpha_{j}$ real, $\alpha_{j}>-1 / 2$, is a classical fact. Moreover, the coefficients $\varkappa_{k}^{2}=D_{k} / D_{k+1}$ are regular functions of all $\alpha_{j}$ (as follows from the determinantal representation for $D_{k}[32,8]$ ). For all complex $\alpha_{j}$ in any fixed closed bounded set of the half-plane $\Re \alpha_{j}>-1 / 2, j=1, \ldots, m$ (denote the set of such $m$-tuples $\left\{\alpha_{j}\right\}_{j=1}^{m}$ by $\widetilde{\Omega}$ ), we shall prove below the existence of a solution to the Riemann-Hilbert problem for all $n$ larger than some $n_{0}>0$. Its asymptotics will be explicitly constructed. We shall also see that the coefficients $\varkappa_{k}, k>n_{0}$ are nonzero and finite for all such $\alpha_{j}$. For $k \leq n_{0}$, the coefficients $\varkappa_{k}^{2}$ are regular and nonzero (as follows from the determinantal representation) and $D_{k}$ are nonzero outside of a possible subset $\hat{\Omega}$ of $\widetilde{\Omega}$. When all $\alpha_{j}$ except for $\alpha_{j_{0}}$ (for some $j_{0}$ ) are fixed provided only $\left\{\alpha_{j}\right\}_{j=1}^{m} \in \widetilde{\Omega}$, we denote the set of values of $\alpha_{j_{0}}$ such that $\left\{\alpha_{j}\right\}_{j=1}^{m} \in \hat{\Omega}$ by $\Omega\left(\alpha_{1}, \ldots, \alpha_{j_{0}-1}, *, \alpha_{j_{0}+1}, \ldots, \alpha_{m}\right)$. As a consequence of the determinantal representation, the system of the orthogonal polynomials exists, and the formula (9) holds for $\alpha_{j_{0}}$ in the $j_{0}$-component of $\widetilde{\Omega}$, outside the set $\Omega\left(\alpha_{1}, \ldots, \alpha_{j_{0}-1}, *, \alpha_{j_{0}+1}, \ldots, \alpha_{m}\right)$. Throughout Section 3 (and, hence, in the differential identity obtained there) we assume that $\left\{\alpha_{j}\right\}_{j=1}^{m} \notin \hat{\Omega}$. (There is no such condition on $\alpha_{j}$ in Section 4.) This provides in Section 5 a proof of Theorem 1 for $\left\{\alpha_{j}\right\}_{j=1}^{m}$ outside the set $\hat{\Omega}$. However, as we shall see below, the error term in the asymptotics of $D_{n}$ is uniform for all $\left\{\alpha_{j}\right\}_{j=1}^{m} \in \widetilde{\Omega}$. Theorem 1 will follow then by continuity.

\section{Differential identity}

Throughout this section, we consider $n$ a fixed positive integer and $\left\{\alpha_{j}\right\}_{j=1}^{m} \in \widetilde{\Omega} \backslash \hat{\Omega}$ (see Section 2). Let us fix some $\nu$ from 1 to $m$, and denote $\alpha_{\nu}=\alpha$. The orthogonality property of the polynomials $p_{n}(x)=\varkappa_{n} x^{n}+\cdots$ implies that

$$
\int_{-\infty}^{\infty} p_{k}(x) x^{j} w(x) d x=\frac{\delta_{j k}}{\varkappa_{j}}, \quad j=0,1, \ldots, k, \quad k=0,1,2, \ldots
$$

Using (91) and this relation, we have

$$
\begin{aligned}
& \frac{d}{d \alpha} \ln D_{n}\left(\alpha_{1}, \ldots, \alpha_{m}\right)=\frac{d}{d \alpha} \ln \prod_{j=0}^{n-1} \varkappa_{j}^{-2}=-2 \sum_{j=0}^{n-1} \frac{\varkappa_{j, \alpha}^{\prime}}{\varkappa_{j}}=-2 \sum_{j=0}^{n-1} \int_{-\infty}^{\infty} p_{j}(x) p_{j, \alpha}^{\prime}(x) w(x) d x= \\
& -\int_{-\infty}^{\infty}\left(\sum_{j=0}^{n-1} p_{j}^{2}(x)\right)_{\alpha}^{\prime} w(x) d x .
\end{aligned}
$$

Here the prime and the lower index $\alpha$ stand for the derivative w.r.t. $\alpha$. Below we also use derivatives w.r.t. $x$ denoting them with the prime and the lower index $x$.

Note that $p_{n}(x)$ are analytic functions of $\alpha_{j}$ as follows, e.g., from their representation as a determinant and regularity of the leading coefficients for $\left\{\alpha_{j}\right\}_{j=1}^{m} \in \widetilde{\Omega} \backslash \hat{\Omega}$. 
By the well-known Christoffel-Darboux formula (e.g., [32]),

$$
\sum_{j=0}^{n-1} p_{j}^{2}(x)=b_{n-1}\left(p_{n, x}^{\prime}(x) p_{n-1}(x)-p_{n}(x) p_{n-1, x}^{\prime}(x)\right)
$$

where $b_{j}$ are coefficients in the recurrence relation for our polynomials:

$$
b_{j-1} p_{j-1}(x)+\left(a_{j}-x\right) p_{j}(x)+b_{j} p_{j+1}(x)=0, \quad j=1,2, \ldots
$$

Let us fix the notation for the 3 leading coefficients of the polynomials $p_{n}(z)$ as follows:

$$
p_{n}(z) \varkappa_{n}^{-1}=z^{n}+\beta_{n} z^{n-1}+\gamma_{n} z^{n-2}+\cdots
$$

Comparing the coefficients at the powers $z^{n+1}, z^{n}$, and $z^{n-1}$ in the recurrence relation, we obtain the following identities we shall use later on:

$$
b_{j}=\frac{\varkappa_{j}}{\varkappa_{j+1}}, \quad a_{j}=\beta_{j}-\beta_{j+1}, \quad\left(\frac{\varkappa_{j-1}}{\varkappa_{j}}\right)^{2}=\gamma_{j}-\gamma_{j+1}-\beta_{j}^{2}+\beta_{j} \beta_{j+1} .
$$

Substituting (15) into (14), differentiating it w.r.t. $\alpha$, using the orthogonality and the above expression for $b_{n-1}$, we obtain:

$$
\begin{aligned}
& \frac{d}{d \alpha} \ln D_{n}\left(\alpha_{1}, \ldots, \alpha_{m}\right)=-n \frac{\varkappa_{n-1, \alpha}^{\prime}}{\varkappa_{n-1}}+\frac{\varkappa_{n-1}}{\varkappa_{n}}\left(J_{1}-J_{2}\right), \\
& J_{1}=\int_{-\infty}^{\infty} p_{n, \alpha}^{\prime}(x) p_{n-1, x}^{\prime}(x) w(x) d x, \quad J_{2}=\int_{-\infty}^{\infty} p_{n, x}^{\prime}(x) p_{n-1, \alpha}^{\prime}(x) w(x) d x .
\end{aligned}
$$

Note that since (17) contains polynomials with indices $n-1$ and $n$ only, it is already an identity one could use to obtain an asymptotic expression for the logarithmic derivative. This identity is valid for a general weight $w(x)$. However, to obtain an asymptotic expression for $(d / d \alpha) \ln D_{n}$, one would have to integrate asymptotics of the polynomials over the whole real line, which would make the calculation rather cumbersome. We shall now see that the particular structure of our weight $w(x)$ allows us to simplify (17) considerably and reduce it to an identity involving only some polynomial coefficients and the values of polynomials and their Cauchy transforms at the points $\mu_{j}$.

Let us evaluate $J_{1}$. Choose points $c_{k}, d_{k}$ according to the inequalities:

$$
-\infty=c_{1}<\mu_{1}<d_{1}=c_{2}<\mu_{2}<d_{2}=c_{3}<\cdots<d_{m-1}=c_{m}<\mu_{m}<d_{m}=\infty
$$

For a small $\varepsilon>0$, split the integral into $3 m$ ones over the intervals $\left(c_{k}, \mu_{k}-\varepsilon\right),\left(\mu_{k}-\varepsilon, \mu_{k}+\varepsilon\right)$, $\left(\mu_{k}+\varepsilon, d_{k}\right)$. The middle ones can be written in the form

$$
\int_{\mu_{k}-\varepsilon}^{\mu_{k}+\varepsilon}\left|x-\mu_{k}\right|^{2 \alpha_{k}} f(x) d x
$$


where $f(x)$ is a smooth function on $\left(\mu_{k}-\varepsilon, \mu_{k}+\varepsilon\right)$. Hence, this integral is of order $O\left(\varepsilon^{2 \alpha_{k}+1}\right)$, small for $\Re \alpha_{k}>-1 / 2$. For the same reason

$$
\left.f(x)\left|x-\mu_{k}\right|^{2 \alpha_{k}}\right|_{\mu_{k}-\varepsilon} ^{\mu_{k}+\varepsilon}=\left(f\left(\mu_{k}+\varepsilon\right)-f\left(\mu_{k}-\varepsilon\right)\right) \varepsilon^{2 \alpha_{k}}=O\left(\varepsilon^{2 \alpha_{k}+1}\right) .
$$

Now we can integrate each of the remaining integrals

$$
\int_{c_{k}}^{\mu_{k}-\varepsilon} p_{n, \alpha}^{\prime}(x) p_{n-1, x}^{\prime}(x) w(x) d x+\int_{\mu_{k}+\varepsilon}^{d_{k}} p_{n, \alpha}^{\prime}(x) p_{n-1, x}^{\prime}(x) w(x) d x
$$

by parts and then take the limit $\varepsilon \rightarrow 0$ to obtain $J_{1}$. Since $w(x)$ decreases as $e^{-x^{2}}$ at infinity and

$$
w(x)_{x}^{\prime}=\left(\sum_{j=1}^{m} \frac{2 \alpha_{j}}{x-\mu_{j}}-2 x\right) w(x),
$$

integration by parts gives (we again use the orthogonality to write $\int p_{n-1} p_{n, x, \alpha}^{\prime \prime} w d x=$ $\left.n \varkappa_{n, \alpha}^{\prime} / \varkappa_{n-1}\right)$

$$
J_{1}=-n \frac{\varkappa_{n, \alpha}^{\prime}}{\varkappa_{n-1}}-2 \sum_{j=1}^{m} \alpha_{j} \int_{-\infty}^{\infty} p_{n-1}(x) \frac{p_{n, \alpha}^{\prime}(x)}{x-\mu_{j}} w(x) d x+2 \int_{-\infty}^{\infty} p_{n-1}(x) p_{n, \alpha}^{\prime}(x) x w(x) d x
$$

where the first $m$ integrals are taken in the principal value sense. Note that they converge for $\Re \alpha_{j}>-1 / 2$. This can be seen directly as follows. Take the $j$ 's one and write it in the form

$$
\int_{-\infty}^{\infty} \frac{\left|x-\mu_{j}\right|^{2 \alpha}}{x-\mu_{j}} f(x) d x=\lim _{\varepsilon \rightarrow 0}\left(\int_{-\infty}^{\mu_{j}-\varepsilon}-\left(\mu_{j}-x\right)^{2 \alpha_{j}-1} f(x) d x+\int_{\mu_{j}+\varepsilon}^{\infty}\left(x-\mu_{j}\right)^{2 \alpha_{j}-1} f(x) d x\right),
$$

where $f(x)$ is a smooth function at $x=\mu_{j}$. Change the variables $y=\mu_{j}-x$ in the first, and $y=x-\mu_{j}$ in the second integral on the r.h.s. Then the sum reduces to

$$
\int_{\varepsilon}^{\infty} y^{2 \alpha_{j}-1}\left(f\left(\mu_{j}+y\right)-f\left(\mu_{j}-y\right)\right) d y .
$$

The integrand is of order $O\left(y^{2 \alpha_{j}}\right)$ for small $y$, and therefore the integral converges as $\varepsilon \rightarrow 0$.

We now simplify the integral. Adding and subtracting $p_{n, \alpha}^{\prime}\left(\mu_{j}\right)$ gives:

$$
\begin{aligned}
& \int_{-\infty}^{\infty} p_{n-1}(x) \frac{p_{n, \alpha}^{\prime}(x)}{x-\mu_{j}} w(x) d x= \\
& \int_{-\infty}^{\infty} p_{n-1}(x) \frac{p_{n, \alpha}^{\prime}(x)-p_{n, \alpha}^{\prime}\left(\mu_{j}\right)}{x-\mu_{j}} w(x) d x+p_{n, \alpha}^{\prime}\left(\mu_{j}\right) \int_{-\infty}^{\infty} \frac{p_{n-1}(x) w(x)}{x-\mu_{j}} d x .
\end{aligned}
$$

The ratio in the first integral on the r.h.s. is obviously a polynomial of degree $n-1$ in $x-\mu_{j}$ with the leading coefficient $\varkappa_{n, \alpha}^{\prime}$. Therefore, by the orthogonality,

$$
\int_{-\infty}^{\infty} p_{n-1}(x) \frac{p_{n, \alpha}^{\prime}(x)-p_{n, \alpha}^{\prime}\left(\mu_{j}\right)}{x-\mu_{j}} w(x) d x=\frac{\varkappa_{n, \alpha}^{\prime}}{\varkappa_{n-1}} .
$$


The last integral in the expression (20) for $J_{1}$ can be rewritten as follows:

$$
\int_{-\infty}^{\infty} p_{n-1}(x)\left(\varkappa_{n, \alpha}^{\prime} x^{n+1}+\left(\varkappa_{n} \beta_{n}\right)_{\alpha}^{\prime} x^{n}+\left(\varkappa_{n} \gamma_{n}\right)_{\alpha}^{\prime} x^{n-1}+\cdots\right) w(x) d x
$$

Expanding here $x^{n+1}$ and $x^{n}$ in terms of the polynomials $p_{j}(x)$, we obtain by the orthogonality and (16) that this integral equals

$$
\frac{\varkappa_{n}}{\varkappa_{n-1}}\left[\frac{\varkappa_{n, \alpha}^{\prime}}{\varkappa_{n}}\left(\frac{\varkappa_{n-1}}{\varkappa_{n}}\right)^{2}+\gamma_{n, \alpha}^{\prime}-\beta_{n} \beta_{n, \alpha}^{\prime}\right] \text {. }
$$

We, therefore, finally obtain

$$
\begin{aligned}
& J_{1}=-\left(n+2 \sum_{j=1}^{m} \alpha_{j}\right) \frac{\varkappa_{n, \alpha}^{\prime}}{\varkappa_{n-1}}-2 \sum_{j=1}^{m} \alpha_{j} p_{n, \alpha}^{\prime}\left(\mu_{j}\right) \int_{-\infty}^{\infty} \frac{p_{n-1}(x) w(x)}{x-\mu_{j}} d x+ \\
& 2 \frac{\varkappa_{n}}{\varkappa_{n-1}}\left[\frac{\varkappa_{n, \alpha}^{\prime}}{\varkappa_{n}}\left(\frac{\varkappa_{n-1}}{\varkappa_{n}}\right)^{2}+\gamma_{n, \alpha}^{\prime}-\beta_{n} \beta_{n, \alpha}^{\prime}\right] .
\end{aligned}
$$

The analysis of $J_{2}$ is similar (and simpler). We obtain that

$$
J_{2}=2 \frac{\varkappa_{n-1, \alpha}^{\prime}}{\varkappa_{n}}-2 \sum_{j=1}^{m} \alpha_{j} p_{n-1, \alpha}^{\prime}\left(\mu_{j}\right) \int_{-\infty}^{\infty} \frac{p_{n}(x) w(x)}{x-\mu_{j}} d x
$$

again with the principal value integral.

Note that at $\alpha_{j}=0$ in the above two equations the terms multiples of $\alpha_{j}$ disappear.

Thus, by (17), (22),$(23)$, and (12), we can now write our identity in terms of the matrix elements of $Y(z)$. Some care is needed when comparing the Cauchy transforms in the second column of $Y(z)$ with the principal value integrals in $J_{1}$ and $J_{2}$ : in particular, note that $Y_{12}(z)$ and $Y_{22}(z)$ are unbounded at $z=\mu_{j}$ if $\Re \alpha_{j}<0$.

Consider $-1 / 2<\Re \alpha_{j} \leq 0, \alpha_{j} \neq 0$. The contribution to the matrix elements $Y_{12}(z)$ and $Y_{22}(z)$ containing a singular part has the form

$$
\int_{c_{j}}^{d_{j}} \frac{\left|x-\mu_{j}\right|^{2 \alpha_{j}}}{x-z} f(x) d x
$$

with a function $f(x)$ analytic in a neighborhood of $x=\mu_{j}$ (analytic continuation off the real axis). On the other hand, the corresponding term in (22) or (23) has the form

$$
\int_{c_{j}}^{d_{j}} \frac{\left|x-\mu_{j}\right|^{2 \alpha_{j}}}{x-\mu_{j}} f(x) d x
$$




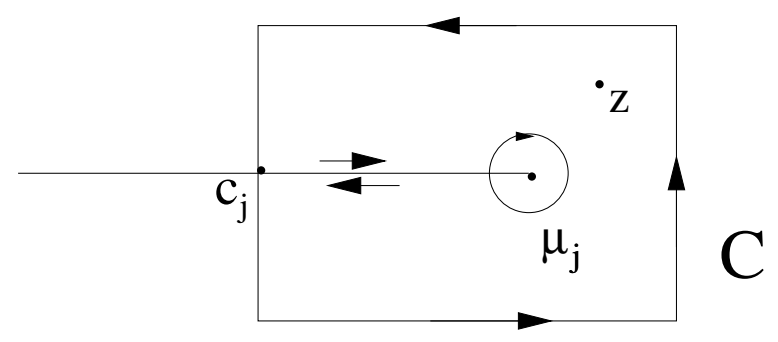

Figure 1: Integration contour for (27).

with the same $f(x)$ and the integral taken in the sense of the principal value. The difference of (24) and (25) can be written as the principal value integral

$$
\left(z-\mu_{j}\right) \lim _{\varepsilon \rightarrow 0}\left(\int_{c_{j}}^{\mu_{j}-\varepsilon} \frac{\left|x-\mu_{j}\right|^{2 \alpha_{j}}}{(x-z)\left(x-\mu_{j}\right)} f(x) d x+\int_{\mu_{j}+\varepsilon}^{d_{j}} \frac{\left|x-\mu_{j}\right|^{2 \alpha_{j}}}{(x-z)\left(x-\mu_{j}\right)} f(x) d x\right) \text {. }
$$

Consider

$$
\int_{C} \frac{\left(x-\mu_{j}\right)^{2 \alpha_{j}}}{(x-z)\left(x-\mu_{j}\right)} f(x) d x
$$

where the contour $C$ is shown in Figure 1.

On the one hand, this integral equals its residue at $x=z$

$$
2 \pi i\left(z-\mu_{j}\right)^{2 \alpha_{j}-1} f(z)
$$

On the other hand, we can rewrite the integral as a sum of parts along the real axes, a circle (of radius $\varepsilon$ ) around $x=\mu_{j}$, and the rest of the contour (denote this rest $C_{0}$ ):

$$
\begin{aligned}
& \int_{C} \frac{\left(x-\mu_{j}\right)^{2 \alpha_{j}}}{(x-z)\left(x-\mu_{j}\right)} f(x) d x=\left(e^{2 \pi i \alpha_{j}}-e^{-2 \pi i \alpha_{j}}\right) \int_{c_{j}}^{\mu_{j}-\varepsilon} \frac{\left|x-\mu_{j}\right|^{2 \alpha_{j}}}{(x-z)\left(x-\mu_{j}\right)} f(x) d x+ \\
& f\left(\mu_{j}\right) \frac{\varepsilon^{2 \alpha_{j}}}{\mu_{j}-z} \frac{e^{-2 \pi i \alpha_{j}}-e^{2 \pi i \alpha_{j}}}{2 \alpha_{j}}+\frac{O\left(\varepsilon^{2 \alpha_{j}+1}\right)}{z-\mu_{j}}+F_{1}(z),
\end{aligned}
$$

where $F_{1}(z)$ is regular as $z \rightarrow \mu_{j}$ and given by the integral over $C_{0}$. The last 2 equations give the first integral in (26):

$$
\begin{aligned}
& \int_{c_{j}}^{\mu_{j}-\varepsilon} \frac{\left|x-\mu_{j}\right|^{2 \alpha_{j}}}{(x-z)\left(x-\mu_{j}\right)} f(x) d x= \\
& \frac{\pi}{\sin 2 \pi \alpha_{j}}\left(z-\mu_{j}\right)^{2 \alpha_{j}-1} f(z)-\frac{\varepsilon^{2 \alpha_{j}}}{2 \alpha_{j}\left(z-\mu_{j}\right)} f\left(\mu_{j}\right)-\frac{F_{1}(z)+O\left(\varepsilon^{2 \alpha_{j}+1}\right) /\left(z-\mu_{j}\right)}{2 i \sin 2 \pi \alpha_{j}} .
\end{aligned}
$$

The second is obtained by a similar analysis with the cut of the root now stretching to the 
right hand side of $\mu_{j}$ and $x-\mu_{j}$ in the numerator replaced by $\left(x-\mu_{j}\right) e^{-i \pi}$. We obtain:

$$
\begin{aligned}
& \int_{\mu_{j}+\varepsilon}^{d_{j}} \frac{\left|x-\mu_{j}\right|^{2 \alpha_{j}}}{(x-z)\left(x-\mu_{j}\right)} f(x) d x= \\
& \frac{-\pi e^{-2 \pi i \alpha_{j}}}{\sin 2 \pi \alpha_{j}}\left(z-\mu_{j}\right)^{2 \alpha_{j}-1} f(z)+\frac{\varepsilon^{2 \alpha_{j}}}{2 \alpha_{j}\left(z-\mu_{j}\right)} f\left(\mu_{j}\right)+\frac{F_{2}(z)+O\left(\varepsilon^{2 \alpha_{j}+1}\right) /\left(z-\mu_{j}\right)}{2 i \sin 2 \pi \alpha_{j}},
\end{aligned}
$$

where $F_{2}(z)$ is regular as $z \rightarrow \mu_{j}$.

Now (26) can be written as follows (note, firstly, that $\varepsilon^{2 \alpha_{j}}$-contributions from the small circles around $\mu_{j}$ from the 2 integrals cancel each other, and secondly, that $F_{1,2}(z)\left(z-\mu_{j}\right)=$ $\left.O\left(z-\mu_{j}\right)\right)$ :

$$
\begin{aligned}
& \int_{c_{j}}^{d_{j}} \frac{\left|x-\mu_{j}\right|^{2 \alpha_{j}}}{x-z} f(x) d x-\int_{c_{j}}^{d_{j}} \frac{\left|x-\mu_{j}\right|^{2 \alpha_{j}}}{x-\mu_{j}} f(x) d x= \\
& e^{-i \pi \alpha_{j}} \frac{\pi i}{\cos \pi \alpha_{j}}\left(z-\mu_{j}\right)^{2 \alpha_{j}} f\left(\mu_{j}\right)+O\left(\left(z-\mu_{j}\right)^{2 \alpha_{j}+1}\right),
\end{aligned}
$$

as $z \rightarrow \mu_{j}, \Im z>0,-1 / 2<\Re \alpha_{j} \leq 0, \alpha_{j} \neq 0$. Thus, the difference of $Y_{j 2}(z), j=1,2$ and the principal value integrals has a nonvanishing part at $\mu_{j}$ given by the first term in the r.h.s. of this equation. The difference vanishes for $\Re \alpha_{j}>0$ (as a similar analysis shows, this difference has only $\left(z-\mu_{j}\right)^{m} \ln \left(z-\mu_{j}\right)$-type singular terms with $m \geq 2 k+1$ for $\alpha_{j}=k+1 / 2$, $k=0,1, \ldots, z \rightarrow \mu_{j}$, and no irregular terms for other positive values of $\left.\alpha_{j}\right)$. Finally, there is a constant difference for $\alpha_{j}=0$.

We denote by $Y^{\mathrm{vp}}\left(\mu_{j}\right)$ the matrix $Y$ with the integrals of the second column replaced by the principal value integrals. Namely,

$$
Y_{k 2}^{\mathrm{vp}}\left(\mu_{j}\right)=\lim _{z \rightarrow \mu_{j}}\left(Y_{k 2}(z)-S_{k}(z)\right)
$$

where the limit is taken along a non-tangential to the real line path in $\mathbb{C}_{+}$, and

$$
S_{k}(z)= \begin{cases}e^{-i \pi \alpha_{j}} \frac{\pi i}{\cos \pi \alpha_{j}}\left(z-\mu_{j}\right)^{2 \alpha_{j}} f_{k}\left(\mu_{j}\right), & \text { for }-1 / 2<\Re \alpha_{j} \leq 0, \alpha_{j} \neq 0 \\ \pi i f_{k}\left(\mu_{j}\right), & \text { for } \alpha_{j}=0 \\ 0, & \text { for } \Re \alpha_{j}>0\end{cases}
$$

with

$$
\begin{aligned}
& f_{1}\left(\mu_{j}\right)=\left(2 \pi i \varkappa_{n}\right)^{-1} p_{n}\left(\mu_{j}\right) \prod_{s \neq j}\left|\mu_{j}-\mu_{s}\right|^{2 \alpha_{s}} \exp \left(-\mu_{j}^{2}\right), \\
& f_{2}\left(\mu_{j}\right)=-\varkappa_{n-1} p_{n-1}\left(\mu_{j}\right) \prod_{s \neq j}\left|\mu_{j}-\mu_{s}\right|^{2 \alpha_{s}} \exp \left(-\mu_{j}^{2}\right) .
\end{aligned}
$$


We then have by (17), (22), (23)

$\frac{d}{d \alpha} \ln D_{n}\left(\alpha_{1}, \ldots, \alpha_{m}\right)=-n\left(\ln \varkappa_{n-1}\right)_{\alpha}^{\prime}-\left(n+2 \sum_{j=1}^{m} \alpha_{j}\right)\left(\ln \varkappa_{n}\right)_{\alpha}^{\prime}-2\left(\frac{\varkappa_{n-1}}{\varkappa_{n}}\right)^{2}\left(\ln \frac{\varkappa_{n-1}}{\varkappa_{n}}\right)_{\alpha}^{\prime}+$

$2 \sum_{j=1}^{m} \alpha_{j}\left(\varkappa_{n}^{-1}\left(\varkappa_{n} Y_{11}\left(\mu_{j}\right)\right)_{\alpha}^{\prime} Y_{22}^{\mathrm{vp}}\left(\mu_{j}\right)-\varkappa_{n-1}\left(\varkappa_{n-1}^{-1} Y_{21}\left(\mu_{j}\right)\right)_{\alpha}^{\prime} Y_{12}^{\mathrm{vp}}\left(\mu_{j}\right)\right)+2\left[\gamma_{n, \alpha}^{\prime}-\beta_{n} \beta_{n, \alpha}^{\prime}\right]$.

This can be somewhat simplified by writing out the derivatives of $\varkappa_{n} Y_{11}\left(\mu_{j}\right), \varkappa_{n-1}^{-1} Y_{21}\left(\mu_{j}\right)$, and using the identity $1=\operatorname{det} Y(\mu)=\operatorname{det} Y^{\mathrm{vp}}(\mu)=Y_{11}(\mu) Y_{22}^{\mathrm{vp}}(\mu)-Y_{21}(\mu) Y_{12}^{\mathrm{vp}}(\mu)$.

We obtain then, recalling that $\alpha=\alpha_{\nu}$, the following

Proposition. Let $\alpha_{j}, \Re \alpha_{j}>-1 / 2, j=1, \ldots, m$ be such that the system of orthogonal polynomials $p_{k}(z)$ with finite nonzero leading coefficients satisfying (8) exists. Fix $n>1$. Let $p_{n}=\varkappa_{n}\left(x^{n}+\beta_{n} x^{n-1}+\gamma_{n} x^{n-2}+\cdots\right)$, the matrix $Y$ be given by (12), and $Y^{\mathrm{vp}}$ be defined in (31). Then

$$
\begin{aligned}
& \frac{d}{d \alpha_{\nu}} \ln D_{n}\left(\alpha_{1}, \ldots, \alpha_{m}\right)=-\left(n+2 \sum_{j=1}^{m} \alpha_{j}\right)\left(\ln \varkappa_{n} \varkappa_{n-1}\right)_{\alpha_{\nu}}^{\prime}-2\left(\frac{\varkappa_{n-1}}{\varkappa_{n}}\right)^{2}\left(\ln \frac{\varkappa_{n-1}}{\varkappa_{n}}\right)_{\alpha_{\nu}}^{\prime}+ \\
& 2 \sum_{j=1}^{m} \alpha_{j}\left(Y_{11}\left(\mu_{j}\right)_{\alpha_{\nu}}^{\prime} Y_{22}^{\mathrm{vp}}\left(\mu_{j}\right)-Y_{21}\left(\mu_{j}\right)_{\alpha_{\nu}}^{\prime} Y_{12}^{\mathrm{vp}}\left(\mu_{j}\right)+\left(\ln \varkappa_{n} \varkappa_{n-1}\right)_{\alpha_{\nu}}^{\prime} Y_{11}\left(\mu_{j}\right) Y_{22}^{\mathrm{vp}}\left(\mu_{j}\right)\right)+ \\
& 2\left[\gamma_{n, \alpha_{\nu}}^{\prime}-\beta_{n} \beta_{n, \alpha_{\nu}}^{\prime}\right], \quad \nu=1, \ldots, m .
\end{aligned}
$$

Note that $\varkappa_{n-1}^{2}=\lim _{z \rightarrow \infty} \frac{i Y_{21}(z)}{2 \pi z^{n-1}}$ and $\gamma_{n}, \beta_{n}$ are expressed similarly. Thus we can find the r.h.s. of (34) asymptotically as $n \rightarrow \infty$ provided the asymptotics of $Y(z)$ are available. These are found in the next section.

\section{Asymptotic analysis of the Riemann-Hilbert prob- lem}

In this section we are guided by [27, 12] where the necessary steepest-descent analysis of the problem (a)-(d) of Section 2 was carried out. We are now faced only with a straightforward, although cumbersome, calculation. We shall see that in order to obtain asymptotics of the r.h.s. of (34) to the needed accuracy, we have to compute the first two terms in the asymptotics of the coefficients $\varkappa_{n}, \beta_{n}, \gamma_{n}$; and only the main term in the asymptotics of $Y\left(\mu_{j}\right)$. In this section we assume only the condition $\Re \alpha_{j}>-1 / 2$ for $\alpha_{j}$.

For the rest of the paper, we assume that

$$
\lambda_{j} \in(-1,1), \quad j=1, \ldots, m, \quad \lambda_{j} \neq \lambda_{k} \quad \text { if } \quad j \neq k .
$$




\section{1 $U, T$, and $S$ transformations of the Riemann-Hilbert problem}

As usual, we perform a series of transformations of the initial problem for $Y(z)$. The first one $Y \rightarrow U$ is a scaling:

$$
Y(z \sqrt{2 n})=(2 n)^{n \sigma_{3} / 2} U(z), \quad \sigma_{3}=\left(\begin{array}{cc}
1 & 0 \\
0 & -1
\end{array}\right) .
$$

The second one $U \rightarrow T$ is given by the formula

$$
U(z)=(2 n)^{\mathcal{A} \sigma_{3} / 2} e^{n l \sigma_{3} / 2} T(z) e^{n(g(z)-l / 2) \sigma_{3}}(2 n)^{-\mathcal{A} \sigma_{3} / 2},
$$

where

$$
\begin{aligned}
& \mathcal{A}=\sum_{j=1}^{m} \alpha_{j}, \quad l=-1-2 \ln 2, \\
& g(z)=\int_{-1}^{1} \ln (z-s) \psi(s) d s, \quad z \in \mathbb{C} \backslash(-\infty, 1], \quad \psi(z)=\frac{2}{\pi} \sqrt{1-z^{2}} .
\end{aligned}
$$

Below we always take the principal branch of the logarithm and roots. The function $\psi(z)$ is the scaled asymptotic density of zeros of $p_{n}(z)$. (Note that $\int_{-1}^{1} \psi(x) d x=1$.) The factor $e^{n g(z)}$ can therefore be regarded as a rough approximation for the polynomials. The function $g(z)$ has the following useful properties:

$$
\begin{aligned}
& g_{+}(x)+g_{-}(x)-2 x^{2}-l=0, \quad \text { for } \quad x \in(-1,1) \\
& g_{+}(x)+g_{-}(x)-2 x^{2}-l<0, \quad \text { for } \quad x \in \mathbb{R} \backslash[-1,1] \\
& g_{+}(x)-g_{-}(x)= \begin{cases}2 \pi i, & \text { for } x \leq-1 \\
2 \pi i \int_{x}^{1} \psi(y) d y, & \text { for } x \in[-1,1] \\
0, & \text { for } x \geq 1\end{cases}
\end{aligned}
$$

From the Riemann-Hilbert problem for $Y(z)$ we obtain the following problem for $T(z)$ :

(a) $T(z)$ is analytic for $z \in \mathbb{C} \backslash \mathbb{R}$.

(b) The boundary values of $T(z)$ are related by the jump condition

$$
\begin{aligned}
& T_{+}(x)=T_{-}(x)\left(\begin{array}{cc}
e^{-n\left(g_{+}(x)-g_{-}(x)\right)} & \prod_{j=1}^{m}\left|x-\lambda_{j}\right|^{2 \alpha_{j}} \\
0 & e^{n\left(g_{+}(x)-g_{-}(x)\right)}
\end{array}\right), \quad x \in(-1,1) \backslash \cup_{j=1}^{m}\left\{\lambda_{j}\right\} . \\
& T_{+}(x)=T_{-}(x)\left(\begin{array}{cc}
1 & \prod_{j=1}^{m}\left|x-\lambda_{j}\right|^{2 \alpha_{j}} e^{n\left(g_{+}(x)+g_{-}(x)-2 x^{2}-l\right)} \\
0 & 1
\end{array}\right), \quad x \in \mathbb{R} \backslash[-1,1] .
\end{aligned}
$$

(c) $T(z)=I+O(1 / z)$ as $z \rightarrow \infty$. 


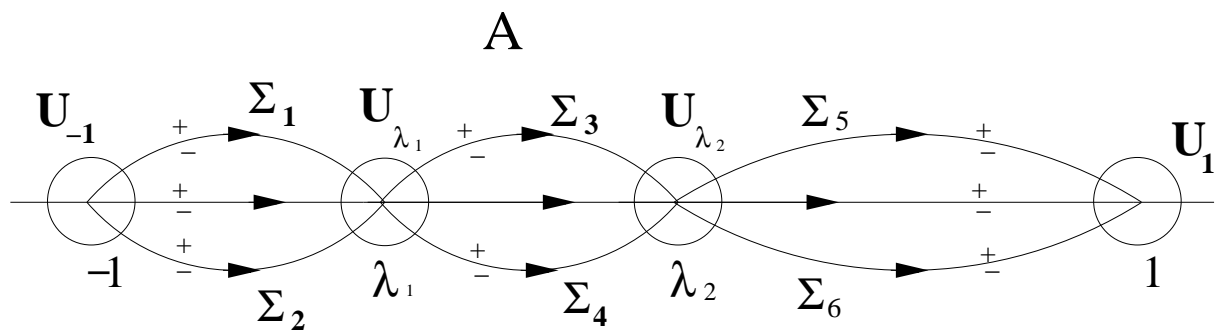

Figure 2: Contour for the Riemann-Hilbert problem $(m=2)$.

(d) Behavior of $T(z)$ as $z \rightarrow \lambda_{j}$ is the same as for $Y(\sqrt{2 n} z)$.

Note that this problem is normalized to 1 at infinity, and the jump matrix on $(-\infty,-1) \cup$ $(1, \infty)$ is exponentially close to the identity (see (38) $)$. We have to exclude small neighborhoods of the points -1 and 1 , where $g_{+}(x)+g_{-}(x)-2 x^{2}-l$ is close to zero, to have a uniform bound.

Now let $h(z)$ be the analytic continuation of

$$
h(x)=g_{+}(x)-g_{-}(x)=2 \pi e^{3 i \pi / 2} \int_{1}^{x} \psi(y) d y
$$

to $\mathbb{C} \backslash((-\infty,-1] \cup[1, \infty))$. Then a simple analysis shows that $\Re h(z)>0$ for $\Im z>0$, and $\Re h(z)<0$ for $\Im z<0$ in some neighborhood of $(-1,1)$. We again exclude neighborhoods of the points -1 and 1 for a uniform estimate.

It turns out it is possible to make use of this fact and split the contour on $(-1,1)$ as shown in Figure 2., transforming again the Riemann-Hilbert problem accordingly (steepest descent method of Deift and Zhou). Then more elements of the jump matrix become exponentially small in $n$, and hence asymptotically negligible, and what remains can be reduced to matrices with constant (in $z$ ) elements. These jump matrices are different in the $m+3$ neighborhoods $U_{ \pm 1}, U_{\lambda_{j}}$ (discs around the points \pm 1 and $\lambda_{j}$ of some sufficiently small fixed radius $\delta$ ), and $U_{\infty}=\mathbb{C} \backslash\left(\overline{U_{1} \cup U_{-1} \cup_{j=1}^{m} U_{\lambda_{j}}}\right)$. Note that in $U_{\lambda_{j}}$ we will have to add an additional cut roughly perpendicular to the real axis (because of nonanalyticity of the absolute value). Our task is then to construct approximate solutions (parametrices) $P_{ \pm 1}(z), P_{\lambda_{j}}(z), P_{\infty}(z)$ in the corresponding neighborhoods and match them on the boundaries $\partial U_{ \pm 1}, \partial U_{\lambda_{j}}$. The solution in $U_{\infty}$ is given in terms of elementary functions; in $U_{ \pm 1}$, in terms of Airy functions [12]; and in $U_{\lambda_{j}}$, in terms of Bessel functions [27, 34]. The "matching" is performed using the known asymptotics of these functions at large arguments and one more " $R$ " transformation of the Riemann-Hilbert problem. This allows us to compute any number of terms in the asymptotics of $Y(z)$.

We start by splitting the contour $(-1,1)$ into lenses (Figure 2). Define an analytic 
continuation of $\prod_{j=1}^{m}\left|x-\mu_{j}\right|^{2 \alpha_{j}}$ as follows:

$$
\omega(z)= \begin{cases}\prod_{j=1}^{m}\left(\lambda_{j}-z\right)^{2 \alpha_{j}}, & \text { for } \Re z<\lambda_{1}, \\ \prod_{j=1}^{\nu}\left(z-\lambda_{j}\right)^{2 \alpha_{j}} \prod_{j=\nu+1}^{m}\left(\lambda_{j}-z\right)^{2 \alpha_{j}}, & \text { for } \lambda_{\nu}<\Re z<\lambda_{\nu+1}, \nu=1, \ldots, m-1 \\ \prod_{j=1}^{m}\left(z-\lambda_{j}\right)^{2 \alpha_{j}}, & \text { for } \Re z>\lambda_{m} .\end{cases}
$$

Splitting of the contour is possible because of the following factorization property of the jump matrix on $(-1,1)$ :

$$
\left(\begin{array}{cc}
e^{-n h(x)} & \omega(x) \\
0 & e^{n h(x)}
\end{array}\right)=\left(\begin{array}{cc}
1 & 0 \\
\omega(x)^{-1} e^{n h(x)} & 1
\end{array}\right)\left(\begin{array}{cc}
0 & \omega(x) \\
-\omega(x)^{-1} & 0
\end{array}\right)\left(\begin{array}{cc}
1 & 0 \\
\omega(x)^{-1} e^{-n h(x)} & 1
\end{array}\right) .
$$

Define a new transformation of our matrix-valued function as follows:

$$
S(z)= \begin{cases}T(z), & \text { for } z \text { outside the lenses, } \\
T(z)\left(\begin{array}{cc}
1 & 0 \\
-\omega(z)^{-1} e^{-n h(z)} & 1
\end{array}\right), & \text { for } z \text { in the upper part of the lenses, } \\
T(z)\left(\begin{array}{cc}
1 & 0 \\
\omega(z)^{-1} e^{n h(z)} & 1
\end{array}\right), & \text { for } z \text { in the lower part of the lenses. }\end{cases}
$$

(Note that we had to contract the lenses at $\lambda_{j}$ because we want $S(z)$ to be analytic in each part of the lenses.)

Then the Riemann-Hilbert problem for $S(z)$ is the following:

(a) $S(z)$ is analytic for $z \in \mathbb{C} \backslash \Sigma$, where $\Sigma=\mathbb{R} \cup \cup_{j=1}^{2 m+2} \Sigma_{j}$.

(b) The boundary values of $S(z)$ are related by the jump condition

$$
S_{+}(x)=S_{-}(x)\left(\begin{array}{cc}
1 & 0 \\
\omega(x)^{-1} e^{\mp n h(x)} & 1
\end{array}\right), \quad x \in \cup_{j=1}^{2 m+2} \Sigma_{j},
$$

where the plus sign in the exponent is on $\Sigma_{2 j}$, and minus, on $\Sigma_{2 j-1}, j=1,2, \ldots, m+1$,

$$
\begin{aligned}
& S_{+}(x)=S_{-}(x)\left(\begin{array}{cc}
0 & \omega(x) \\
-\omega(x)^{-1} & 0
\end{array}\right), \quad x \in(-1,1) \backslash \cup_{j=1}^{m}\left\{\lambda_{j}\right\} . \\
& S_{+}(x)=S_{-}(x)\left(\begin{array}{cc}
1 & \omega(x) e^{n\left(g_{+}(x)+g_{-}(x)-2 x^{2}-l\right)} \\
0 & 1
\end{array}\right), \quad x \in \mathbb{R} \backslash[-1,1] .
\end{aligned}
$$

(c) $S(z)=I+O(1 / z)$ as $z \rightarrow \infty$.

(d) For $\Re \alpha_{j} \leq 0$, the matrix function $S(z)$ has the following behavior as $z \rightarrow \lambda_{j}$ :

$$
S(z)=O\left(\begin{array}{cc}
1 & \left|z-\lambda_{j}\right|^{2 \alpha_{j}} \\
1 & \left|z-\lambda_{j}\right|^{2 \alpha_{j}}
\end{array}\right), \quad \text { as } z \rightarrow \lambda_{j}, z \in \mathbb{C} \backslash \Sigma .
$$


For $\Re \alpha_{j}>0$, the matrix function $S(z)$ has the following behavior as $z \rightarrow \lambda_{j}$ :

$$
S(z)=\left\{\begin{array}{cc}
O\left(\begin{array}{cc}
1 & 1 \\
1 & 1
\end{array}\right), & \text { as } z \rightarrow \lambda_{j} \text { from outside the lenses, } \\
O\left(\begin{array}{ll}
\left|z-\lambda_{j}\right|^{-2 \alpha_{j}} & 1 \\
\left|z-\lambda_{j}\right|^{-2 \alpha_{j}} & 1
\end{array}\right), & \text { as } z \rightarrow \lambda_{j} \text { from inside the lenses. }
\end{array}\right.
$$

Recalling the remarks above, we see that, outside the neighborhoods $U_{\lambda_{j}}, U_{ \pm 1}$, the jump matrix on $\Sigma_{j}, j=1, \ldots, 2 m+2$ is uniformly exponentially close to the identity.

Let us now start constructing parametrices which give (in their respective regions) the leading contribution to the asymptotics.

\subsection{Parametrix in $U_{\infty}$}

First, we expect the following problem for the parametrix in $U_{\infty}$ :

(a) $P_{\infty}(z)$ is analytic for $z \in \mathbb{C} \backslash[-1,1]$,

(b) with the jump condition on $(-1,1)$

$$
P_{\infty,+}(x)=P_{\infty,-}(x)\left(\begin{array}{cc}
0 & \omega(x) \\
-\omega(x)^{-1} & 0
\end{array}\right), \quad x \in(-1,1) \backslash \cup_{j=1}^{m}\left\{\lambda_{j}\right\},
$$

(c) and the following behavior at infinity

$$
P_{\infty}(z)=I+O\left(\frac{1}{z}\right), \quad \text { as } z \rightarrow \infty
$$

A solution $P_{\infty}(z)$ can be found in the same way as in [28].

$$
P_{\infty}(z)=\frac{1}{2}\left(\mathcal{D}_{\infty}\right)^{\sigma_{3}}\left(\begin{array}{cc}
a+a^{-1} & -i\left(a-a^{-1}\right) \\
i\left(a-a^{-1}\right) & a+a^{-1}
\end{array}\right) \mathcal{D}(z)^{-\sigma_{3}}, \quad a(z)=\left(\frac{z-1}{z+1}\right)^{1 / 4}
$$

where the cut of the root is the interval $(-1,1)$. (Note that $\operatorname{det} P_{\infty}(z)=1$.) Here

$$
\mathcal{D}(z)=\exp \left[\frac{\sqrt{z^{2}-1}}{2 \pi} \int_{-1}^{1} \frac{\ln \omega(\xi)}{\sqrt{1-\xi^{2}}} \frac{d \xi}{z-\xi}\right], \quad \mathcal{D}_{\infty}=\lim _{z \rightarrow \infty} \mathcal{D}(z)
$$

The Szegö function $\mathcal{D}(z)$ is analytic outside the interval $[-1,1]$ with the boundary values satisfying $\mathcal{D}_{+}(x) \mathcal{D}_{-}(x)=\omega(x), x \in(-1,1) \backslash \cup_{j=1}^{m}\left\{\lambda_{j}\right\}$. We can calculate the integral in (50) directly as follows. First, replace $\omega(x)$ by $\omega(\nu x)$; second, take the derivative of the integral w.r.t. $\nu$. Set then $\xi=\cos \theta$, and integrate in the complex plane of $e^{i \theta}$. After that integrate the 
result w.r.t. $\nu$ over $(0,1)$, and finally, use the value of the integral at $\nu=0$. This procedure is actually a simple "scalar" analogue of the whole present work. For $\lambda_{j} \in(-1,1)$, we obtain

$$
\mathcal{D}(z)=\left(z+\sqrt{z^{2}-1}\right)^{-\mathcal{A}} \prod_{j=1}^{m}\left(z-\lambda_{j}\right)^{\alpha_{j}}, \quad \mathcal{A}=\sum_{j=1}^{m} \alpha_{j}
$$

Therefore,

$$
\mathcal{D}_{\infty}=2^{-\mathcal{A}}
$$

\subsection{Parametrices at $z=\lambda_{j}$}

Let us now construct the parametrices in $U_{\lambda_{j}}, j=1, \ldots, m$. The construction is the same for every $j$. We look for an analytic matrix-valued function $P_{\lambda_{j}}(z)$ in a neighborhood of $U_{\lambda_{j}}$ which satisfies the same jump conditions as $S(z)$ on $\Sigma \cap U_{\lambda_{j}}$, has the same behavior as $z \rightarrow \lambda_{j}$, and (instead of a condition at infinity) satisfies the matching condition

$$
P_{\lambda_{j}}(z) P_{\infty}^{-1}(z)=I+O(1 / n)
$$

uniformly on the boundary $\partial U_{\lambda_{j}}$ as $n \rightarrow \infty$.

Using the analytic continuation of $\psi(y)$, define:

$$
\phi(z)= \begin{cases}h(z) / 2=e^{3 i \pi / 2} \pi \int_{1}^{z} \psi(y) d y, & \text { for } \Im z>0 \\ e^{-i \pi} h(z) / 2=e^{i \pi / 2} \pi \int_{1}^{z} \psi(y) d y, & \text { for } \Im z<0\end{cases}
$$

It is easy to verify that the function $e^{\phi(z)}$ is analytic outside $[-1,1]$. Note that $h(z)$ was defined analytic outside $\mathbb{R} \backslash(-1,1)$. Set furthermore,

$$
\hat{f}(z)=\pi \int_{\lambda_{j}}^{z} \psi(y) d y
$$

Let us now choose the exact form of the cuts $\Sigma$ in $U_{\lambda_{j}}$ so that their images under the mapping $\zeta=n \hat{f}(z)$ are direct lines (Figure 3). Note that $\zeta(z)=n \hat{f}(z)$ is analytic and one-to-one in the neighborhood of $U_{\lambda_{j}}$, and it takes the real axis to the real axis. We have:

$$
\zeta=n \hat{f}(z)=n 2 \sqrt{1-\lambda_{j}^{2}}\left(z-\lambda_{j}\right)\left(1+O\left(z-\lambda_{j}\right)\right), \quad z \rightarrow \lambda_{j}
$$

Set

$$
W_{j}(z)=\prod_{i=1}^{j-1}\left(z-\lambda_{i}\right)^{\alpha_{i}} \prod_{i=j+1}^{m}\left(\lambda_{i}-z\right)^{\alpha_{i}} \times \begin{cases}\left(z-\lambda_{j}\right)^{\alpha_{j}}, & \text { if } \pi / 2<|\arg \hat{f}(z)|<\pi \\ \left(\lambda_{j}-z\right)^{\alpha_{j}}, & \text { if } 0<|\arg \hat{f}(z)|<\pi / 2\end{cases}
$$

This function has the following jumps on $\Gamma_{3}, \Gamma_{7}$ :

$$
W_{j+}(z)=W_{j-}(z) e^{i \pi \alpha_{j}}, \quad \zeta \in \Gamma_{3} \cup \Gamma_{7} .
$$


Moreover, the functions $W_{j}(z)$ and $\omega(z)$ are related in $U_{\lambda_{j}}$ as follows:

$$
W_{j}^{2}(z)=\omega(z) e^{-2 \pi i \alpha_{j}}
$$

in the region $\Re \zeta>0, \Im z>0 \cap \Im \zeta>0$, and in the region $\Re \zeta<0, \Im z<0 \cap \Im \zeta<0$; whereas

$$
W_{j}^{2}(z)=\omega(z) e^{2 \pi i \alpha_{j}}
$$

in the region $\Re \zeta<0, \Im z>0 \cap \Im \zeta>0$, and in the region $\Re \zeta>0, \Im z<0 \cap \Im \zeta<0$. Note that by definition of $\omega(z), \arg \omega(z)=0$ on $\Im z=0$, and $\omega(z)$ is continuous through this line. Hence the function $W_{j}(z)$ also has jumps on $\Gamma_{1}, \Gamma_{5}$ given by the formulae:

$$
W_{j+}(z)=W_{j-}(z) \times\left\{\begin{array}{ll}
e^{-2 \pi i \alpha_{j}}, & \text { for } \zeta \in \Gamma_{1} \\
e^{2 \pi i \alpha_{j}}, & \text { for } \zeta \in \Gamma_{5}
\end{array} .\right.
$$

We look for $P_{\lambda_{j}}(z)$ in the form:

$$
P_{\lambda_{j}}(z)=E_{n}(z) P^{(1)}(z) W_{j}(z)^{-\sigma_{3}} e^{-n \phi(z) \sigma_{3}},
$$

where $E_{n}(z)$ is analytic and invertible in the neighborhood of $U_{\lambda_{j}}$, and therefore does not affect the jump and analyticity conditions. It is needed and so chosen that the matching condition be satisfied.

It is easy to verify that $P^{(1)}(z)$ satisfies jump conditions with constant jump matrices. Because of $W_{j}(z), P^{(1)}(z)$ has also an additional jump condition along the line $\Re \hat{f}(z)=0$ in $U_{\lambda_{j}}$. Set

$$
P^{(1)}(z)=\Psi_{\alpha_{j}}(\zeta)=\Psi_{\alpha_{j}}(n \hat{f}(z)),
$$

where the cuts for $\Psi_{\alpha_{j}}(\zeta)$ are shown in Figure 3, and the Riemann Hilbert problem for it is as follows:

(a) $\Psi_{\alpha_{j}}$ is analytic for $\zeta \in \mathbb{C} \backslash \cup_{j=1}^{8} \Gamma_{j}$.

(b) $\Psi_{\alpha_{j}}$ satisfies the following jump conditions:

$$
\begin{aligned}
& \Psi_{\alpha_{j},+}(\zeta)=\Psi_{\alpha_{j},-}(\zeta)\left(\begin{array}{cc}
0 & 1 \\
-1 & 0
\end{array}\right), \quad \text { for } \zeta \in \Gamma_{1} \cup \Gamma_{5}, \\
& \Psi_{\alpha_{j},+}(\zeta)=\Psi_{\alpha_{j},-}(\zeta)\left(\begin{array}{cc}
1 & 0 \\
e^{-2 \pi i \alpha_{j}} & 1
\end{array}\right), \quad \text { for } \zeta \in \Gamma_{2} \cup \Gamma_{6}, \\
& \Psi_{\alpha_{j},+}(\zeta)=\Psi_{\alpha_{j},-}(\zeta) e^{\pi i \alpha_{j} \sigma_{3}}, \quad \text { for } \zeta \in \Gamma_{3} \cup \Gamma_{7}, \\
& \Psi_{\alpha_{j},+}(\zeta)=\Psi_{\alpha_{j},-}(\zeta)\left(\begin{array}{cc}
1 & 0 \\
e^{2 \pi i \alpha_{j}} & 1
\end{array}\right), \quad \text { for } \zeta \in \Gamma_{4} \cup \Gamma_{8} .
\end{aligned}
$$




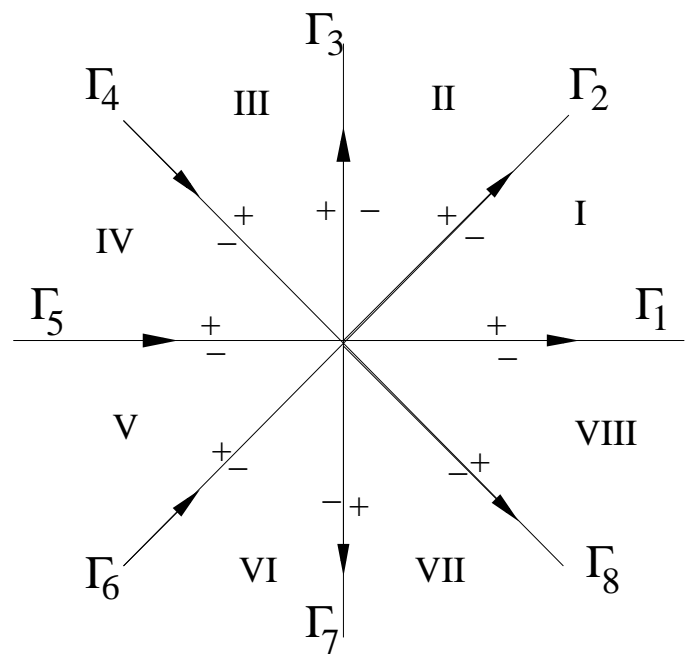

Figure 3: The auxiliary contour for the parametrix at $\lambda_{j}$.

(c) For $\Re \alpha_{j} \leq 0$ the matrix function $\Psi_{\alpha_{j}}(\zeta)$ has the following behavior as $\zeta \rightarrow 0$ :

$$
\Psi_{\alpha_{j}}(\zeta)=O\left(\begin{array}{ll}
|\zeta|^{\alpha_{j}} & |\zeta|^{\alpha_{j}} \\
|\zeta|^{\alpha_{j}} & |\zeta|^{\alpha_{j}}
\end{array}\right), \quad \zeta \rightarrow 0
$$

For $\Re \alpha_{j}>0$ the matrix function $\Psi_{\alpha_{j}}(\zeta)$ has the following behavior as $\zeta \rightarrow 0$ :

$$
\Psi_{\alpha_{j}}(\zeta)=\left\{\begin{array}{c}
O\left(\begin{array}{cc}
|\zeta|^{\alpha_{j}} & |\zeta|^{-\alpha_{j}} \\
|\zeta|^{\alpha_{j}} & |\zeta|^{-\alpha_{j}}
\end{array}\right), \quad \text { as } z \rightarrow \lambda_{j} \text { with } \zeta \in \text { II, III, VI, VII, } \\
O\left(\begin{array}{cc}
|\zeta|^{-\alpha_{j}} & |\zeta|^{-\alpha_{j}} \\
|\zeta|^{-\alpha_{j}} & |\zeta|^{-\alpha_{j}}
\end{array}\right), \quad \text { as } z \rightarrow \lambda_{j} \text { with } \zeta \in \text { I, IV, V, VIII. }
\end{array}\right.
$$

The solution of this Riemann-Hilbert problem was constructed in [34] in terms of Bessel functions. For our purposes, we need its explicit form only in the region $I I$ (see Figure 3 ). There, we have (see [34]):

$$
\Psi_{\alpha_{j}}(\zeta)=\sqrt{\pi \zeta}\left(\begin{array}{cc}
I_{\alpha_{j}+1 / 2}\left(\zeta e^{-i \pi / 2}\right) e^{-i \pi \alpha_{j} / 2} & -\frac{1}{\pi} K_{\alpha_{j}+1 / 2}\left(\zeta e^{-i \pi / 2}\right) e^{i \pi \alpha_{j} / 2} \\
-i I_{\alpha_{j}-1 / 2}\left(\zeta e^{-i \pi / 2}\right) e^{-i \pi \alpha_{j} / 2} & -\frac{i}{\pi} K_{\alpha_{j}-1 / 2}\left(\zeta e^{-i \pi / 2}\right) e^{i \pi \alpha_{j} / 2}
\end{array}\right), \quad \zeta \in I I
$$

where $I_{\beta}(x)$ and $K_{\beta}(x)$ are modified Bessel functions (see [1]). The solution in other regions can be reproduced by applying the jump conditions to (170).

Taking also $E_{n}(z)$ from [27, 34, and substituting all into (62), we obtain for $z \in z(I I)$, where $z(I I)$ is the image of $I I$ under the mapping $\zeta \rightarrow z$,

$$
P_{\lambda_{j}}(z)=P_{\infty}(z) W_{j}(z)^{\sigma_{3}} e^{\left(n \phi_{+}\left(\lambda_{j}\right)+\alpha_{j} \pi i / 2\right) \sigma_{3}} e^{-i \pi \sigma_{3} / 4} \frac{1}{\sqrt{2}}\left(\begin{array}{cc}
1 & i \\
i & 1
\end{array}\right) \Psi_{\alpha_{j}}(n \hat{f}(z)) W_{j}(z)^{-\sigma_{3}} e^{-n \phi(z) \sigma_{3}} .
$$


The argument of Bessel functions is uniformly large on $\partial U_{\lambda_{j}}$. Substituting asymptotics of Bessel functions for large arguments

$$
\Psi_{\alpha_{j}}(\zeta)=\frac{1}{\sqrt{2}}\left(\begin{array}{cc}
1 & -i \\
-i & 1
\end{array}\right)\left[I+\frac{i}{4 \zeta}\left(\begin{array}{cc}
-2 \alpha_{j}^{2} & -2 i \alpha_{j} \\
-2 i \alpha_{j} & 2 \alpha_{j}^{2}
\end{array}\right)+O\left(\zeta^{-2}\right)\right] e^{\left(\pi / 4-\alpha_{j} \pi / 2-\zeta\right) i \sigma_{3}}, \zeta \in I I,
$$

(and similar ones for other regions (see [27, 34])) into (71) (and its analogues for other regions), we can verify that the matching condition is satisfied. For that we need only the main asymptotic term plus the error term. The fit is ensured by the choice of $E_{n}(z)$. The exponential factor in the asymptotics of Bessel functions cancels with $e^{-n \phi(z) \sigma_{3}}$ in (71) leaving a constant in $z$ factor. (Note that $\hat{f}(z)=i \phi(z)-i \phi_{+}\left(\lambda_{j}\right)$.) Moreover, considering further terms, we can extend (53) into full asymptotic series in inverse powers of $n$. For our calculations we need to know explicitly the first correction term:

$$
\begin{aligned}
& P_{\lambda_{j}}(z) P_{\infty}^{-1}(z)=I+\Delta_{1}(z)+O\left(1 / n^{2}\right), \\
& \Delta_{1}(z)=-P_{\infty}(z) W_{j}(z)^{\sigma_{3}} e^{\left(n \phi_{+}\left(\lambda_{j}\right)+\alpha_{j} \pi i / 2-\pi i / 4\right) \sigma_{3}} \frac{i}{2 \zeta}\left(\begin{array}{cc}
\alpha_{j}^{2} & i \alpha_{j} \\
i \alpha_{j} & -\alpha_{j}^{2}
\end{array}\right) \times \\
& W_{j}(z)^{-\sigma_{3}} e^{-\left(n \phi_{+}\left(\lambda_{j}\right)+\alpha_{j} \pi i / 2-\pi i / 4\right) \sigma_{3}} P_{\infty}^{-1}(z), \\
& \quad z \in \partial z(I I),
\end{aligned}
$$

where $\partial z(I I)$ is the part of $\partial U_{\lambda_{j}}$ whose $\zeta$-image is in $I I$. As the calculation for the other regions shows, this expression for $\Delta_{1}(z)$ extends by analytic continuation to the whole boundary $\partial U_{\lambda_{j}}$ (cf. [27, 34]). Moreover, it gives a meromorphic function in a neighborhood of $U_{\lambda_{j}}$ with a simple pole at $z=\lambda_{j}$. The error term $O\left(1 / n^{2}\right)$ in (73) is uniform on $\partial U_{\lambda_{j}}$.

Note that the absence of jumps for $\operatorname{det} \Psi(\zeta)$ and the fact that $\Re \alpha_{j}>-1 / 2$ implies that the only possible singularity of $\operatorname{det} \Psi(\zeta)$ (at $\zeta=0$ ) is removable. Thus, $\operatorname{det} \Psi(\zeta)$ is analytic. Moreover, the asymptotics of Bessel functions give that $\operatorname{det} \Psi(\zeta) \rightarrow 1$ as $\zeta \rightarrow \infty$, which implies that $\operatorname{det} \Psi(\zeta) \equiv 1$. Using this, we easily deduce from (71) that also $\operatorname{det} P_{\lambda_{j}}(z) \equiv 1$.

Note that it follows from the asymptotics of Bessel functions and the fact that

$$
D(z) W_{j}(z)^{-1}=\left(z+\sqrt{z^{2}-1}\right)^{-\mathcal{A}} e^{ \pm i \pi \sum_{k=j^{\prime}}^{m} \alpha_{k}}
$$

(where $j^{\prime}=j$ or $j^{\prime}=j+1$ and the sign is different in different quadrants of the $\zeta$-plane), that all the terms (including the error term) are uniform for $\alpha_{k}$ in a bounded set of the half-plane $\Re \alpha_{k}>-1 / 2$. This observation (and similar ones for $\partial U_{ \pm 1}$ ) will be very important below.

\subsection{Parametrices at $z= \pm 1$}

Now let us construct parametrices in the remaining regions $U_{ \pm 1}$. These are obtained by a slight generalization of the results of [12] (which can be viewed as the case $\omega(z)=1$ ). We are looking for an analytic matrix-valued function in $U_{1}$ which has the same jump relation as $S(z)$ there and satisfies the matching condition on the boundary:

$$
P_{1}(z) P_{\infty}^{-1}(z)=I+O(1 / n) .
$$


The solution is:

$P_{1}=E(z) Q(\zeta) e^{-n \phi(z) \sigma_{3}} \omega(z)^{-\sigma_{3} / 2}, \quad E(z)=P_{\infty}(z) \omega(z)^{\sigma_{3} / 2} e^{i \pi \sigma_{3} / 4} \sqrt{\pi}\left(\begin{array}{cc}1 & -1 \\ 1 & 1\end{array}\right) \zeta^{\sigma_{3} / 4} e^{-\pi i / 12}$

and $Q(\zeta)$ is given by the expression (7.9) of [12] in terms of Airy functions (in the notation of [12] $\left.Q(\zeta)=\Psi^{\sigma}(\zeta)\right)$. In these formulas

$$
\zeta(z)=\left(\frac{3}{2} n e^{-i \pi} \phi(z)\right)^{2 / 3}
$$

As is easy to verify, so defined $\zeta(z)$ is an analytic function in a neighborhood of $z=1$ (the cut of the square root is the interval $(-1,1))$, and

$$
\zeta(z)=2 n^{2 / 3}(z-1)\left(1+\frac{1}{10}(z-1)+O\left((z-1)^{2}\right)\right)
$$

The argument of Airy function on $\partial U_{1}$ is uniformly large, so we can expand it into the asymptotic series and proceed the same way as for $\partial U_{\lambda_{j}}$. As a result we have the matching condition (75) extended to the full asymptotic expansion in inverse powers of $n$. We shall need below only the first 2 terms:

$$
\begin{aligned}
& P_{1}(z) P_{\infty}^{-1}(z)=I+\Delta_{1}(z)+O\left(1 / n^{2}\right) \\
& \Delta_{1}(z)=P_{\infty}(z) \omega(z)^{\sigma_{3} / 2} e^{\pi i \sigma_{3} / 4} \frac{1}{12}\left(\begin{array}{cc}
1 / 6 & 1 \\
-1 & -1 / 6
\end{array}\right) e^{-\pi i \sigma_{3} / 4} \omega(z)^{-\sigma_{3} / 2} P_{\infty}^{-1}(z) \frac{3}{2} \zeta^{-3 / 2}, \\
& z \in \partial U_{1}
\end{aligned}
$$

The function $\Delta_{1}(z)$ is meromorphic in the neighborhood of $U_{1}$ with a second order pole at $z=1$.

The argument for the parametrix in $U_{-1}$ is similar. We just mention the solution:

$$
\begin{aligned}
& P_{-1}=E(z) \sigma_{3} Q\left(e^{-i \pi} \zeta\right) \sigma_{3} e^{-n \tilde{\phi}(z) \sigma_{3}} \omega(z)^{-\sigma_{3} / 2} \\
& E(z)=P_{\infty}(z) \omega(z)^{\sigma_{3} / 2} e^{i \pi \sigma_{3} / 4} \sqrt{\pi}\left(\begin{array}{cc}
1 & 1 \\
-1 & 1
\end{array}\right)\left(e^{-i \pi} \zeta\right)^{\sigma_{3} / 4} e^{-\pi i / 12}
\end{aligned}
$$

Here

$$
\zeta(z)=e^{-i \pi}\left(\frac{3}{2} n \tilde{\phi}(z)\right)^{2 / 3}, \quad \tilde{\phi}(z)=\left\{\begin{array}{ll}
h(z) / 2-i \pi=e^{3 i \pi / 2} \pi \int_{-1}^{z} \psi(y) d y, & \text { for } \Im z>0 \\
e^{i \pi} h(z) / 2+i \pi=e^{5 i \pi / 2} \pi \int_{-1}^{z} \psi(y) d y, & \text { for } \Im z<0
\end{array} .\right.
$$

For $z \rightarrow-1$ :

$$
\zeta(z)=2 n^{2 / 3}(1+z)\left(1-\frac{1}{10}(1+z)+O\left((1+z)^{2}\right)\right)
$$




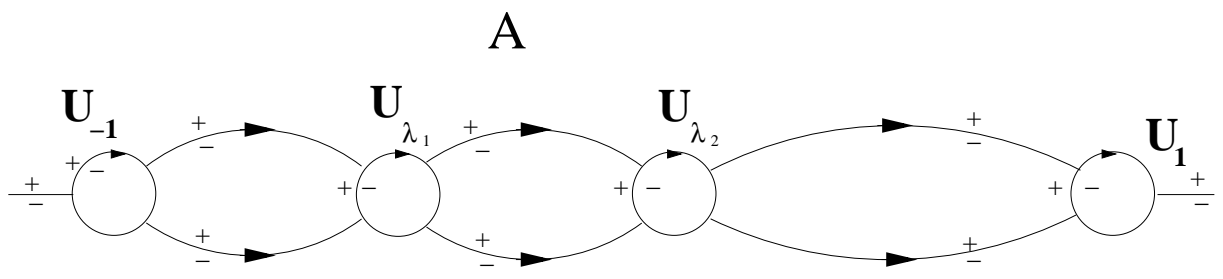

Figure 4: Contour for the $R$-Riemann-Hilbert problem $(m=2)$.

The first 2 terms in the matching condition are as follows:

$$
\begin{aligned}
& P_{-1}(z) P_{\infty}^{-1}(z)=I+\Delta_{1}(z)+O\left(1 / n^{2}\right) \\
& \Delta_{1}(z)=P_{\infty}(z) \omega(z)^{\sigma_{3} / 2} e^{\pi i \sigma_{3} / 4} \frac{1}{12}\left(\begin{array}{cc}
1 / 6 & -1 \\
1 & -1 / 6
\end{array}\right) e^{-\pi i \sigma_{3} / 4} \omega(z)^{-\sigma_{3} / 2} P_{\infty}^{-1}(z) \frac{3}{2}\left(e^{-i \pi} \zeta\right)^{-3 / 2} \\
& z \in \partial U_{-1}
\end{aligned}
$$

As in (173), the error term in (179) (resp., (83)) is uniform for all $z \in \partial U_{1}$ (resp., $z \in \partial U_{-1}$ ) and $\alpha_{j}$ 's in a bounded set.

\subsection{Final transformation of the problem}

Now the construction of the parametrices is complete, and we are ready for the final transformation of the Riemann-Hilbert problem. Let

$$
R(z)= \begin{cases}S(z) P_{\infty}^{-1}(z), & z \in U_{\infty} \backslash \Sigma \\ S(z) P_{\lambda_{j}}^{-1}(z), \quad z \in U_{\lambda_{j}} \backslash \Sigma, j=1, \ldots, m \\ S(z) P_{1}^{-1}(z), \quad z \in U_{1} \backslash \Sigma \\ S(z) P_{-1}^{-1}(z), \quad z \in U_{-1} \backslash \Sigma\end{cases}
$$

It is easily seen that this function has jumps only on $\partial U_{ \pm 1}, \partial U_{\lambda_{j}}$, and parts of $\Sigma_{j}, \mathbb{R} \backslash[-1,1]$ lying outside the neighborhoods $U_{ \pm 1}, U_{\lambda_{j}}$ (we denote these parts without the end-points $\Sigma^{\text {out}}$ ). The contour is shown in Figure 4 (for $m=2$ ). Outside of it, $R(z)$ is analytic: Indeed, as follows from (45), (46), (68), (69), and the fact that $\operatorname{det} P_{\lambda_{j}}=1$, the function $S(z) P_{\lambda_{j}}^{-1}(z)$, for example, has at most a singularity of order less than 1 at $\lambda_{j}$, which implies, due to the absence of jumps, that $S(z) P_{\lambda_{j}}^{-1}(z)$ is analytic in $U_{\lambda_{j}}$. The argument for other regions is similar.

Note that $R(z)=I+O(1 / z)$ as $z \rightarrow \infty$. 
The jumps are as follows:

$$
\begin{aligned}
& R_{+}(x)=R_{-}(x) P_{\infty}(x)\left(\begin{array}{cc}
1 & \omega(x) e^{n\left(g_{+}(x)+g_{-}(x)-2 x^{2}-l\right)} \\
0 & 1
\end{array}\right) P_{\infty}(x)^{-1}, \quad x \in \mathbb{R} \backslash[-1-\delta, 1+\delta], \\
& R_{+}(x)=R_{-}(x) P_{\infty}(x)\left(\begin{array}{cc}
1 & 0 \\
\omega(x)^{-1} e^{\mp n h(x)} & 1
\end{array}\right) P_{\infty}(x)^{-1}, \quad x \in \Sigma_{k}^{\text {out }}, \quad k=1, \ldots, 2 m+2,
\end{aligned}
$$

where the plus sign in the exponent is on $\Sigma_{2 j}^{\text {out }}$, and minus, on $\Sigma_{2 j-1}^{\text {out }}, j=1, \ldots, m+1$,

$R_{+}(x)=R_{-}(x) P_{\lambda_{j}}(x) P_{\infty}(x)^{-1}, \quad x \in \partial U_{\lambda_{j}} \backslash\{$ intersection points $\}, \quad j=1, \ldots, m$, $R_{+}(x)=R_{-}(x) P_{ \pm 1}(x) P_{\infty}(x)^{-1}, \quad x \in \partial U_{ \pm 1} \backslash\{$ intersection points $\}$.

Here $\delta$ is the radius of $U_{1}, U_{-1}$.

The jump matrix on $\Sigma^{\text {out }}$ can be estimated uniformly in $\alpha$ as $I+O(\exp (-\varepsilon n|x|))$, where $\varepsilon$ is a positive constant. The jump matrices on $\partial U_{\lambda_{j}, \pm 1}$ admit a uniform expansion in inverse powers of $n$ (the first 2 terms of which are given by (73), (79), and (83)):

$$
I+\Delta_{1}(z)+\Delta_{2}(z)+\ldots+\Delta_{k}(z)+O\left(n^{-k-1}\right) .
$$

Every $\Delta_{j}$ is of order $1 / n^{j}$. (The above expressions give us an explicit form of $\Delta_{1}(z)$ in each of the neighborhoods.)

We look for $R(z)$ asymptotically in the form $R(z) \sim R_{0}(z)+R_{1}(z)+R_{2}(z)+\cdots$, where $R_{j}(z), j>0$, is of the same order as $\Delta_{j}$. It can be shown following Theorems 7.8-7.10 of [12] that for any $k \geq 1$

$$
R(z)=\sum_{j=0}^{k} R_{j}(z)+O\left(n^{-k-1}\right), \quad R_{0}=I,
$$

uniformly for all $z$ and for $\alpha_{j}$ in a bounded set of the half-plane $\Re \alpha_{j}>-1 / 2, j=1, \ldots, m$. The set can extend up to the boundary $\Re \alpha_{j}=-1 / 2$.

Moreover, we can substitute this asymptotic expansion into (85) and, collecting the terms of the same order, obtain:

$$
\begin{aligned}
& R_{0+}(x)+R_{1+}(x)+\cdots \sim\left(R_{0-}(x)+R_{1-}(x)+\cdots\right)\left(I+\Delta_{1}(x)+\cdots\right), \quad x \in \partial U_{\lambda_{j}, \pm 1} . \\
& R_{0+}(x)=R_{0-}(x) \quad \Rightarrow \quad R_{0}(z)=I \\
& R_{1+}(x)-R_{1-}(x)=\Delta_{1}(x) \\
& R_{2+}(x)-R_{2-}(x)=R_{1-}(x) \Delta_{1}(x)+\Delta_{2}(x), \\
& R_{k+}(x)-R_{k-}(x)=\sum_{j=1}^{k} R_{k-j,-}(x) \Delta_{j}(x), \quad k=1,2, \ldots
\end{aligned}
$$

The main term in the asymptotics of the polynomials is given therefore by the parametrices at the appropriate points $z$. The expressions for $R_{k}(z)$ follow from the Sokhotsky-Plemelj 
formulas:

$$
R_{1}(z)=\frac{1}{2 \pi i} \int_{\partial U} \frac{\Delta_{1}(x) d x}{x-z}, \quad R_{2}(z)=\frac{1}{2 \pi i} \int_{\partial U} \frac{R_{1-}(x) \Delta_{1}(x)+\Delta_{2}(x)}{x-z} d x, \quad \ldots
$$

$\partial U=\partial U_{1} \cup \partial U_{-1} \cup_{j=1}^{m} \partial U_{\lambda_{j}}$. Note that the contours are traversed in the negative direction.

For reader's convenience, we present a variant of the proof of (87 891), a combination of the arguments from [12] and [28]. First, we need a bound on $R(z)$. Let

$$
\Delta \equiv J-I
$$

where $J$ is the jump matrix for $R$ on $\Sigma_{R} \equiv \Sigma^{\text {out }} \cup \partial U$ (see (85)). The jump condition and the behaviour of $R(z)$ at infinity imply that

$$
R(z)=I+C\left(R_{-} \Delta\right), \quad z \in \mathbb{C} \backslash \Sigma_{R},
$$

where

$$
C(f)=\frac{1}{2 \pi i} \int_{\Sigma_{R}} f(s) \frac{d s}{s-z}
$$

is the Cauchy operator on $\Sigma_{R}$. Hence,

$$
R_{-}(s)=I+C_{-}\left(R_{-} \Delta\right),
$$

where $C_{-}(f)=\lim _{z \rightarrow s} C(f)$ as $z$ approaches a point $s \in \Sigma_{R} \backslash\{$ intersection points $\}$ from the - side of $\Sigma_{R}$. It is known that $C_{-}$is a bounded operator from $L^{2}\left(\Sigma_{R}\right)$ to $L^{2}\left(\Sigma_{R}\right)$. Now defining the operator

$$
C_{\Delta}(f)=C_{-}(f \Delta)
$$

we represent (92) in the form

$$
\left(I-C_{\Delta}\right)\left(R_{-}-I\right)=C_{\Delta}(I) .
$$

Since by the estimates above $\Delta(s)=O(1 / n)$ and $\Delta(s)=O(\exp (-\varepsilon n|s|))$ (on $\Sigma^{\text {out }}$ ) for $n \rightarrow \infty$ uniformly in $\alpha_{j}$ 's (in a bounded set), and $s \in \Sigma_{R}$, the operator norm of $C_{\Delta}$ acting on $L^{2}\left(\Sigma_{R}\right),\left\|C_{\Delta}\right\|=O(1 / n)$, and $I-C_{\Delta}$ is invertible by a Neuman series for $n$ sufficiently large. Moreover, $\left\|C_{\Delta}(I)\right\|_{L^{2}\left(\Sigma_{R}\right)}=O(1 / n)$. Thus (93) gives

$$
R_{-}(s)=I+\left(I-C_{\Delta}\right)^{-1}\left(C_{\Delta}(1)\right),
$$

where

$$
\left\|R_{-}(s)-I\right\|_{L^{2}\left(\Sigma_{R}\right)}=O(1 / n) .
$$

Hence, by (91),

$$
R(z)=I+C\left[\Delta+\left(I-C_{\Delta}\right)^{-1}\left(C_{\Delta}(I)\right) \Delta\right],
$$

and a matrix norm for some $\varepsilon_{1}>0$ and $\operatorname{dist}\left(z, \Sigma_{R}\right) \geq \varepsilon_{1}$,

$$
|R(z)-I| \leq|C(\Delta)|+\left|C\left(\left(R_{-}-I\right) \Delta\right)\right| \leq \frac{c_{1}}{n}+c_{2}|| R_{-}(s)-I\left\|_{L^{2}\left(\Sigma_{R}\right)}|| \Delta\right\|_{L^{2}\left(\Sigma_{R}\right)} \leq \frac{c_{3}}{n}
$$




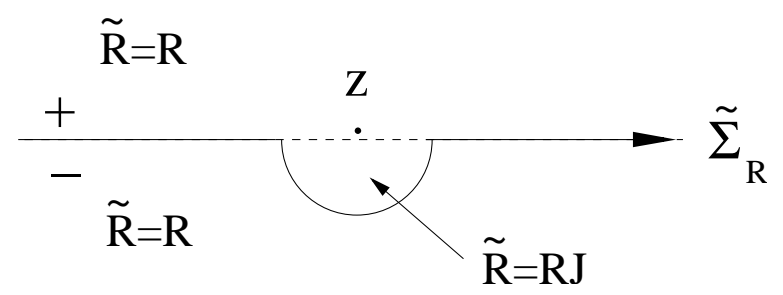

Figure 5: Deformed part of the contour $\Sigma_{R}$.

uniformly in $\alpha$ and $z$ for some $c_{1}, c_{2}, c_{3}>0$, and $n$ larger then some $n_{0}$.

To obtain a uniform estimate for all $z \in \mathbb{C} \backslash \Sigma_{R}$, we (for a $z$ with $\operatorname{dist}\left(z, \Sigma_{R}\right)<\varepsilon_{1}$ ) deform the contour as shown in Figure 5. Here $\tilde{\Sigma}_{R}$ is the same as $\Sigma_{R}$ with the dotted part replaced by the semicircle of radius $\varepsilon_{1} . \tilde{R}(z)$ is defined as shown, and $J$ is the analytic continuation of the jump matrix for $R$ on $\Sigma_{R}$. (It is easy to show that the continuation exists in a neighborhood of the original $\Sigma_{R}$. Neighbourhoods where 2 lines intersect are analyzed similarly.) $\tilde{R}(z)$ satisfies the same Riemann-Hilbert problem as $R(z)$ but on the contour $\tilde{\Sigma}_{R}$. The argument leading to (96) for $R$ and $\Sigma_{R}$ holds for $\tilde{R}$ and $\tilde{\Sigma}_{R}$ as well. Therefore (see Figure 5),

$$
|R(z)-I|=|\tilde{R}(z)-I| \leq c_{3} / n .
$$

Analysis of the analytic continuation $J(z)$ shows that we can find the same $c_{3}$ for all $\alpha_{j}$ 's in a bounded set, $n>n_{0}$, and all $z \in \mathbb{C} \backslash \Sigma_{R}$ up to the boundary $\Sigma_{R}$. This is the estimate we need.

We now proceed with the proof by induction. On $\Sigma^{\text {out }}$, we define $\Delta_{j} \equiv 0$. Consider the function $R_{1}(z)$ analytic outside $\Sigma_{R}$, satisfying the jump condition $R_{1+}(s)-R_{1-}(s)=\Delta_{1}(s)$, $s \in \Sigma_{R}$, and the condition $R_{1}(z)=O(1 / z)$ at infinity. The unique solution of this RiemannHilbert problem is given by the Sokhotsky-Plemelj formula:

$$
R_{1}(z)=C\left(\Delta_{1}\right)=\frac{1}{2 \pi i} \int_{\Sigma_{R}} \Delta_{1}(s) \frac{d s}{s-z} .
$$

Because of the estimate $\Delta_{1}(s)=O(1 / n)$, uniform in $\alpha_{j}$ 's, $s \in \Sigma_{R}$ as $n \rightarrow \infty$, we have

$$
R_{1}(z)=O(1 / n), \quad n \rightarrow \infty,
$$

uniform in $\alpha_{j}$ 's and $z$ satisfying $\operatorname{dist}\left(z, \Sigma_{R}\right) \geq \varepsilon_{1}$. Deforming the contour, we extend this estimate to a uniform one for all $z \in \mathbb{C} \backslash \Sigma_{R}$. By (91) and (99),

$$
R(z)-I-R_{1}(z)=C\left(R_{-} \Delta-\Delta_{1}\right)=C\left(\left(R_{-}-I\right) \Delta+\Delta-\Delta_{1}\right) .
$$

The (uniform in $s \in \Sigma_{R}$ and $\alpha_{j}$ 's) estimates $R_{-}(s)-I=O(1 / n)$ (98)), $\Delta(s)=O(1 / n)$, $\Delta(s)=O(\exp (-\varepsilon n|s|))$ (on $\left.\Sigma^{\text {out }}\right), \Delta(s)-\Delta_{1}(s)=O\left(1 / n^{2}\right)$ (on $\partial U$ ) imply that

$$
R(z)-I-R_{1}(z)=O\left(1 / n^{2}\right), \quad n \rightarrow \infty
$$


uniformly in $\alpha_{j}$ 's, and $z$ such that $\operatorname{dist}\left(z, \Sigma_{R}\right) \geq \varepsilon_{1}$. By a contour deformation argument this result extends uniformly for $z \in \mathbb{C} \backslash \Sigma_{R}$.

Next solving the Riemann-Hilbert problem for $R_{2}$ (i.e., with the jump $R_{2+}-R_{2-}=$ $R_{1-} \Delta_{1}+\Delta_{2}$ ) we get

$$
R_{2}(z)=C\left(R_{1-} \Delta_{1}+\Delta_{2}\right) .
$$

By (100) and the estimates $\Delta_{k}(s)=O\left(1 / n^{k}\right)$, we have as above for $R_{1}$ :

$$
R_{2}(z)=O\left(1 / n^{2}\right), \quad n \rightarrow \infty
$$

uniformly in $\alpha_{j}$ 's, $z \in \mathbb{C} \backslash \Sigma_{R}$. By (98), (102), and the estimates for $\Delta$,

$$
\begin{aligned}
& R(z)-I-R_{1}(z)-R_{2}(z)=C\left(R_{-} \Delta-\Delta_{1}-R_{1-} \Delta_{1}-\Delta_{2}\right)= \\
& C\left(\left(R_{-}-I\right)\left(\Delta-\Delta_{1}\right)\right)+C\left(\Delta-\Delta_{1}-\Delta_{2}\right)+C\left(\left(R_{-}-I-R_{1-}\right) \Delta_{1}\right)=O\left(n^{-3}\right), \\
& n \rightarrow \infty
\end{aligned}
$$

with the same uniformity property in $\alpha_{j}$ 's and $z$.

The general $k$ 'th induction step is carried out similarly. We have, using estimates from the first $k-1$ steps,

$$
R_{k}(z)=C\left(\sum_{j=1}^{k} R_{k-j,-} \Delta_{j}\right)=O\left(n^{-k}\right) .
$$

and for the error after $k$ terms

$$
\begin{aligned}
& R(z)-I-\sum_{j=1}^{k} R_{k}(z)=C\left(R_{-} \Delta-\sum_{j=1}^{k} \sum_{i=1}^{j} R_{j-i,-} \Delta_{i}\right)= \\
& C\left(\left(R_{-}-I\right)\left[\Delta-\sum_{j=1}^{k-1} \Delta_{j}\right]\right)+C\left(\Delta-\left[\sum_{j=1}^{k} \Delta_{j}\right]\right)+ \\
& C\left(\sum_{j=1}^{k-1}\left[R_{-}-I-\sum_{i=1}^{k-j} R_{i-}\right] \Delta_{j}\right)=O\left(n^{-k-1}\right) .
\end{aligned}
$$

The last 2 estimates are valid uniformly for $\alpha_{j}$ 's in a bounded set and $z \in \mathbb{C} \backslash \Sigma_{R}$. This concludes the proof of (8789).

\subsection{Calculation of the asymptotics for the polynomials}

In the intersection of the region $\mathrm{A}$ (the area outside the lenses) and the neighborhoods $U$ we have by (84), (43), (87),

$$
T(z)=S(z)=P_{\theta}(z)+R_{1}(z) P_{\theta}(z)+O\left(1 / n^{2}\right) P_{\theta}(z), \quad z \in U_{\theta} \cap A, \quad \theta= \pm 1, \lambda_{j}, \infty .
$$


It turns out that to prove Theorem 1 using (34), we need to evaluate only $P_{\lambda_{j}}(z)$ as $z \rightarrow \lambda_{j}$ (i.e., the main asymptotic term of $T$ at $\lambda_{j}$ ) and (108) as $z \rightarrow \infty$ (i.e., the first 2 terms of $T$ at infinity).

We evaluate $P_{\lambda_{j}}(z)$ as $z \rightarrow \lambda_{j}$ inside the region $z(I I)$ (see Figure 3 ). $P_{\lambda_{j}}(z)$ there is given by (71), and (70).

Tracing back the transformations (108), (36), and (35), we obtain for the main asymptotic term

$Y(z \sqrt{2 n})=(2 n)^{n \sigma_{3} / 2} e^{n l \sigma_{3} / 2}(2 n)^{\mathcal{A} \sigma_{3} / 2}(I+O(1 / n)) P_{\lambda_{j}}(z)(2 n)^{-\mathcal{A} \sigma_{3} / 2} e^{n(g(z)-l / 2) \sigma_{3}}, \quad z \in z(I I)$.

For application in the identity (34), we need to analyze equation (109) as $z$ approaches $\lambda_{j}$ along a path in $z(I I)$. Note first (recall (51)) that

$$
\begin{aligned}
& \mathcal{D}(z) W_{j}(z)^{-1}=e^{i \pi \tilde{\alpha}_{j}}\left(z+\sqrt{z^{2}-1}\right)^{-\mathcal{A}}=e^{i \pi \tilde{\alpha}_{j}-i \mathcal{A}\left(\pi / 2-\tau_{j}\right)}\left(1+O\left(z-\lambda_{j}\right)\right), \\
& \frac{1}{\sqrt{2}}\left(\sqrt{\lambda_{j}+1}+\sqrt{\lambda_{j}-1_{+}}\right)=e^{i\left(\pi / 2-\tau_{j}\right) / 2}, \quad \tau_{j}=\arcsin \lambda_{j} \quad \tilde{\alpha}_{j}=\sum_{k=j}^{m} \alpha_{k} .
\end{aligned}
$$

Therefore, the product of the first 3 factors in (171) gives as $z \rightarrow \lambda_{j}$ :

$$
\begin{aligned}
& \frac{2^{-\mathcal{A} \sigma_{3}}}{\sqrt{2}\left(1-\lambda_{j}^{2}\right)^{1 / 4}}\left(\begin{array}{cc}
e^{-(\mathcal{A}+1 / 2) \tau_{j} i-\left(2 \tilde{\alpha}_{j}-\mathcal{A}-\alpha_{j}\right) i \pi / 2+n \phi_{+}\left(\lambda_{j}\right)} & e^{(\mathcal{A}+1 / 2) \tau_{j} i+\left(2 \tilde{\alpha}_{j}-\mathcal{A}-\alpha_{j}\right) i \pi / 2-n \phi_{+}\left(\lambda_{j}\right)} \\
-e^{-(\mathcal{A}-1 / 2) \tau_{j} i-\left(2 \tilde{\alpha}_{j}-\mathcal{A}-\alpha_{j}\right) i \pi / 2+n \phi_{+}\left(\lambda_{j}\right)} & e^{(\mathcal{A}-1 / 2) \tau_{j} i+\left(2 \tilde{\alpha}_{j}-\mathcal{A}-\alpha_{j}\right) i \pi / 2-n \phi_{+}\left(\lambda_{j}\right)}
\end{array}\right) \times \\
& \left(1+O\left(z-\lambda_{j}\right)\right) .
\end{aligned}
$$

By (56), we need the asymptotics of Bessel functions at a small argument for (70) (see [1] for them). For $\alpha_{j} \neq 1 / 2+k, k=0,1, \ldots$,

$$
\begin{aligned}
& \Psi_{\alpha_{j}}(\zeta)= \\
& \left.\begin{array}{cc}
C_{1}\left(\alpha_{j}\right) \zeta^{\alpha_{j}+1} & -\frac{1}{\sqrt{2 \pi}}(\zeta / 2)^{-\alpha_{j}} e^{i \frac{\pi}{2}\left(2 \alpha_{j}+1 / 2\right)} \Gamma\left(\alpha_{j}+1 / 2\right)+C_{2}\left(\alpha_{j}\right) \zeta^{\alpha_{j}+1} \\
-i(\zeta / 2)^{\alpha_{j}} e^{-i \frac{\pi}{2}\left(2 \alpha_{j}-1 / 2\right)} \frac{\sqrt{2 \pi}}{\Gamma\left(\alpha_{j}+1 / 2\right)} & C_{3}\left(\alpha_{j}\right) \zeta^{-\alpha_{j}+1}+C_{4}\left(\alpha_{j}\right) \zeta^{\alpha_{j}}
\end{array}\right) \times \\
& (1+O(\zeta)), \quad z \in z(I I),
\end{aligned}
$$

where $C_{j}\left(\alpha_{j}\right)$ are constants whose precise expressions will not be important below. For $\alpha_{j}=1 / 2+k, k=0,1, \ldots$, the constants $C_{1}$ and $C_{3}$ remain the same, while $C_{2}\left(\alpha_{j}\right)$ is replaced with $C_{5}\left(\alpha_{j}\right)+C_{6}\left(\alpha_{j}\right) \ln \zeta$, and $C_{4}\left(\alpha_{j}\right)$ is replaced with $C_{7}\left(\alpha_{j}\right)+C_{8}\left(\alpha_{j}\right) \ln \zeta$.

Now the part of the product of the next 5 factors in (171) with the $\zeta^{2 \alpha_{j}}$ terms omitted ("regularized" part) gives for $z \rightarrow \lambda_{j}$ and $\alpha_{j} \neq 0$ :

$$
\begin{aligned}
& \left(e^{-i \pi \sigma_{3} / 4} \frac{1}{\sqrt{2}}\left(\begin{array}{cc}
1 & i \\
i & 1
\end{array}\right) \Psi_{\alpha_{j}}(n \hat{f}(z)) W_{j}(z)^{-\sigma_{3}}\right)_{\mathrm{reg}} \rightarrow \\
& \left(\begin{array}{cc}
1 & -\frac{1}{2} \\
1 & \frac{1}{2}
\end{array}\right)\left(\frac{\sqrt{\pi}\left(n \sqrt{1-\lambda_{j}^{2}}\right)^{\alpha_{j}}}{\Gamma\left(\alpha_{j}+1 / 2\right)}\right)^{\sigma_{3}} \prod_{k \neq j}\left|\lambda_{k}-\lambda_{j}\right|^{-\alpha_{k} \sigma_{3}} .
\end{aligned}
$$


This result will correspond to the "principal value" part of $Y$ given by (31). The omitted part of order $\zeta^{2 \alpha_{j}}$ does not contribute to the "principal value" part of the total product for $Y$ because all the correction terms are $O(\zeta)$ as $\zeta \rightarrow 0$ and $\Re \alpha_{j}>-1 / 2$.

The expression (113) is also valid for $\alpha_{j}=0$ (since, e.g., (32) for this case can be obtained by letting $\alpha_{j} \rightarrow 0$ in the $\zeta^{2 \alpha_{j}}$ term with $\left.\alpha_{j}<0\right)$.

Finally, the product of the last factors in (171) and (109) gives by (38):

$$
e^{n(g(z)-\phi(z)-l / 2)}=e^{n\left(g_{+}\left(\lambda_{j}\right)-\phi_{+}\left(\lambda_{j}\right)-l / 2\right)}\left(1+O\left(z-\lambda_{j}\right)\right)=e^{n \lambda_{j}^{2} \sigma_{3}}\left(1+O\left(z-\lambda_{j}\right)\right) .
$$

Taking the product of all contributions, we have:

$$
\begin{gathered}
\lim _{z \rightarrow \lambda_{j}} Y^{\mathrm{vp}}(z \sqrt{2 n})=\left(\frac{n}{2 e}\right)^{n \sigma_{3} / 2}(2 n)^{\mathcal{A} \sigma_{3} / 2}(I+O(1 / n)) 2^{-\mathcal{A} \sigma_{3}}\left(1-\lambda_{j}^{2}\right)^{-1 / 4} \times \\
\left(\begin{array}{cc}
\sqrt{2} \cos \frac{1}{2}\left(t_{j}-\tau_{j}\right) & -\frac{i}{\sqrt{2}} \sin \frac{1}{2}\left(t_{j}-\tau_{j}\right) \\
-i \sqrt{2} \sin \frac{1}{2}\left(t_{j}+\tau_{j}\right) & \frac{1}{\sqrt{2}} \cos \frac{1}{2}\left(t_{j}+\tau_{j}\right)
\end{array}\right)\left(\frac{\sqrt{\pi}\left(n \sqrt{1-\lambda_{j}^{2}}\right)^{\alpha_{j}} e^{n \lambda_{j}^{2}}}{(2 n)^{\mathcal{A} / 2} \Gamma\left(\alpha_{j}+1 / 2\right)}\right)^{\sigma_{3}} \prod_{k \neq j}\left|\lambda_{k}-\lambda_{j}\right|^{-\alpha_{k} \sigma_{3}} \\
t_{j}=2 \pi n \int_{\lambda_{j}}^{1} \psi(y) d y+\pi \alpha_{j}-2 \pi \sum_{i=j}^{m} \alpha_{i}+\mathcal{A}\left(\pi-2 \tau_{j}\right), \quad \tau_{j}=\arcsin \lambda_{j} .
\end{gathered}
$$

We now turn to the asymptotics of the coefficients $\varkappa_{n}, \beta_{n}$ and $\gamma_{n}$ of $p_{n}(z)$. Here we need the first 2 asymptotic terms in $n$. As usual, we compute them investigating the limit $z \rightarrow \infty$ of $Y(z)$. Namely, by (12),

$$
\varkappa_{n-1}^{2}=\lim _{z \rightarrow \infty} \frac{i Y_{21}(z)}{2 \pi z^{n-1}}, \quad U_{11}(z)=z^{n}+\frac{\beta_{n}}{\sqrt{2 n}} z^{n-1}+\frac{\gamma_{n}}{2 n} z^{n-2}+\cdots
$$

As $z \rightarrow \infty$, we need to know asymptotics of $Y(z)$ in the region $A$, which are given by the expressions (cf. (109) ) :

$$
\begin{aligned}
& Y(z \sqrt{2 n})=(2 n)^{n \sigma_{3} / 2} U(z), \\
& U(z)=e^{n l \sigma_{3} / 2}(2 n)^{\mathcal{A} \sigma_{3} / 2}\left(I+R_{1}(z)+O\left(1 / n^{2}\right)\right) P_{\infty}(z) e^{n(g(z)-l / 2) \sigma_{3}}(2 n)^{-\mathcal{A} \sigma_{3} / 2}, \\
& z \in A \cap U_{\infty} .
\end{aligned}
$$

Let us compute $R_{1}(z)$ using (89). Consider first the neighborhood $U_{\lambda_{j}}$. Substituting $\Delta_{1}(x)$ given by (73) into (89) and calculating residues at a simple pole $x=\lambda_{j}$, we obtain the contribution to $R_{1}$ from the neighborhood $U_{\lambda_{j}}$ :

$$
\begin{aligned}
& R_{1}^{\left(\lambda_{j}\right)}(z)=\frac{1}{2 \pi i} \int_{\partial U_{\lambda_{j}}} \frac{\Delta_{1} d x}{x-z}=-\frac{1}{z}\left(1+\frac{\lambda_{j}}{z}+O\left(z^{-2}\right)\right) \frac{1}{2 \pi i} \int_{\partial U_{\lambda_{j}}} \Delta_{1} d x= \\
& \frac{1}{z}\left(1+\frac{\lambda_{j}}{z}+O\left(z^{-2}\right)\right) \frac{\mathcal{D}_{\infty}^{\sigma_{3}} \alpha_{j}}{4 n\left(1-\lambda_{j}^{2}\right)}\left(\begin{array}{cc}
-\alpha_{j} \lambda_{j}+\sin t_{j} & i\left(\alpha_{j}-\cos \left(t_{j}-\tau_{j}\right)\right) \\
i\left(\alpha_{j}+\cos \left(t_{j}+\tau_{j}\right)\right) & \alpha_{j} \lambda_{j}-\sin t_{j}
\end{array}\right) \mathcal{D}_{\infty}^{-\sigma_{3}},
\end{aligned}
$$


where $t_{j}, \tau_{j}$ are defined in (116).

To compute the contribution from the neighborhood $U_{1}$, we repeat the calculation now using $\Delta_{1}(z)$ from (79). An additional complication is that we need to calculate residues in the pole $z=1$ of second order. We obtain:

$$
\begin{aligned}
& R_{1}^{(1)}=\frac{1}{2 \pi i} \int_{\partial U_{1}} \frac{\Delta_{1} d x}{x-z}=\frac{1}{z}\left(1+\frac{1}{z}+O\left(z^{-2}\right)\right) \frac{\mathcal{D}_{\infty}^{\sigma_{3}}}{8 n}\left(\begin{array}{cc}
1 / 8-\mathcal{A}^{2} & i / 6+i \mathcal{A}+i \mathcal{A}^{2} \\
i / 6-i \mathcal{A}+i \mathcal{A}^{2} & -1 / 8+\mathcal{A}^{2}
\end{array}\right) \mathcal{D}_{\infty}^{-\sigma_{3}}+ \\
& \frac{1}{z^{2}} \frac{5 \mathcal{D}_{\infty}^{\sigma_{3}}}{8 \cdot 24 n}\left(\begin{array}{cc}
-1 & i \\
i & 1
\end{array}\right) \mathcal{D}_{\infty}^{-\sigma_{3}} .
\end{aligned}
$$

Here the dependence on $\alpha_{j}$ comes from the expansion (see (51)):

$$
\frac{\mathcal{D}^{2}(z)}{\omega(z)}=1-2 \sqrt{2} \mathcal{A} \sqrt{z-1}+4 \mathcal{A}^{2}(z-1)+O\left((z-1)^{3 / 2}\right), \quad \text { as } z \rightarrow 1 .
$$

A similar calculation for $U_{-1}$ gives

$$
\begin{aligned}
& R_{1}^{(-1)}=\frac{1}{2 \pi i} \int_{\partial U_{-1}} \frac{\Delta_{1} d x}{x-z}=\frac{1}{z}\left(1-\frac{1}{z}+O\left(z^{-2}\right)\right) \frac{\mathcal{D}_{\infty}^{\sigma_{3}}}{8 n}\left(\begin{array}{cc}
-1 / 8+\mathcal{A}^{2} & i / 6+i \mathcal{A}+i \mathcal{A}^{2} \\
i / 6-i \mathcal{A}+i \mathcal{A}^{2} & 1 / 8-\mathcal{A}^{2}
\end{array}\right) \mathcal{D}_{\infty}^{-\sigma_{3}}+ \\
& \frac{1}{z^{2}} \frac{5 \mathcal{D}_{\infty}^{\sigma_{3}}}{8 \cdot 24 n}\left(\begin{array}{cc}
-1 & -i \\
-i & 1
\end{array}\right) \mathcal{D}_{\infty}^{-\sigma_{3}} .
\end{aligned}
$$

In this case

$$
\frac{\mathcal{D}^{2}(z)}{\omega(z)}=1+2 \sqrt{2} i \mathcal{A} \sqrt{z+1}-4 \mathcal{A}^{2}(z+1)+O\left((z+1)^{3 / 2}\right), \quad \text { as } z \rightarrow-1 .
$$

Summing up all the contributions (119), (120), and (122), we obtain:

$$
R_{1}=R_{1}^{(1)}+R_{1}^{(-1)}+\sum_{j=1}^{m} R_{1}^{\left(\lambda_{j}\right)}
$$

Substituting this into (118) and using the expansions for $z \rightarrow \infty$ :

$$
\begin{aligned}
& \frac{\mathcal{D}_{\infty}}{\mathcal{D}(z)}=1+\frac{1}{z} \sum_{j=1}^{m} \alpha_{j} \lambda_{j}+\frac{1}{2 z^{2}}\left[\left(\sum_{j=1}^{m} \alpha_{j} \lambda_{j}\right)^{2}+\sum_{j=1}^{m} \alpha_{j} \lambda_{j}^{2}-\frac{1}{2} \mathcal{A}\right]+O\left(z^{-3}\right), \\
& a(z)=1-\frac{1}{2 z}+\frac{1}{8 z^{2}}+O\left(z^{-3}\right), \quad g(z)=\ln z-\frac{1}{8 z^{2}}+O\left(z^{-4}\right),
\end{aligned}
$$

we finally obtain from (117)

$$
\varkappa_{n-1}^{2}=\frac{2^{n-1+\mathcal{A}} n^{-\mathcal{A}}}{\sqrt{\pi}(n-1) !}\left\{1-\frac{1}{2 n}\left(\mathcal{A}^{2}-\mathcal{A}+\sum_{j=1}^{m} \frac{1}{1-\lambda_{j}^{2}}\left[\alpha_{j}^{2}+\alpha_{j} \cos \left(t_{j}+\tau_{j}\right)\right]\right)+O\left(\frac{1}{n^{2}}\right)\right\},
$$




$$
\begin{gathered}
\beta_{n}=\sqrt{2 n}\left\{\sum_{j=1}^{m} \alpha_{j} \lambda_{j}+\frac{1}{4 n} \sum_{j=1}^{m} \frac{\alpha_{j} \sin t_{j}-\alpha_{j}^{2} \lambda_{j}}{1-\lambda_{j}^{2}}+O\left(\frac{1}{n^{2}}\right)\right\}, \\
\gamma_{n}=n\left\{-\frac{n-1}{4}+\left(\sum_{j=1}^{m} \alpha_{j} \lambda_{j}\right)^{2}+\sum_{j=1}^{m} \alpha_{j} \lambda_{j}^{2}-\frac{\mathcal{A}}{2}+\frac{1}{4 n}\left[\mathcal{A}-\mathcal{A}^{2}+\sum_{j=1}^{m} \alpha_{j}^{2}\right.\right. \\
\left.\left.-\sum_{j=1}^{m} \frac{\alpha_{j} \cos \left(t_{j}+\tau_{j}\right)+\alpha_{j}^{2} \lambda_{j}^{2}}{1-\lambda_{j}^{2}}+2 \sum_{j=1}^{m} \frac{\alpha_{j} \sin t_{j}-\alpha_{j}^{2} \lambda_{j}}{1-\lambda_{j}^{2}} \sum_{k=1}^{m} \alpha_{k} \lambda_{k}\right]+O\left(\frac{1}{n^{2}}\right)\right\},
\end{gathered}
$$

where $\tau_{j}=\arcsin \lambda_{j}, \mathcal{A}=\sum_{j=1}^{m} \alpha_{j}$, and $t_{j}$ is defined by (116) and (37). The error terms here and in (115) are uniform for $\alpha_{j}$ in a bounded set provided only $\Re \alpha_{j}>-1 / 2$. Let us track the $\alpha$-dependence of the error terms in more detail. These terms in (126)-(128) are those in (118), at worst multiplied by a polynomial in $\alpha_{j}$ (independent of $n$ ) and $2^{-\mathcal{A}}$ coming from the expansion (125) of $D(z)$. The error term in (115) is the same as the one in (109). Now the error terms in (109) and (118) are those from (87) which we showed to have the above uniformity property.

Thus we constructed a solution to the Riemann-Hilbert problem of Section 2 for $n>n_{0}$, $\alpha_{j}$ in any bounded set of the half-plane $\Re \alpha_{j}>-1 / 2$. By uniqueness, it gives the orthogonal polynomials via (12). On the other hand, the determinantal representation for the orthogonal polynomials shows that $R(z)$ is an analytic function of $\alpha_{j}$ 's. Furthermore, $R_{k}(z)$ are also analytic functions of $\alpha_{j}$ 's by construction. Thus, the error term in (107) is both analytic and uniform in $\alpha_{j}$ 's in a bounded set of the half-plane $\Re \alpha_{j}>-1 / 2$. Therefore, it is differentiable in $\alpha_{j}$ 's (the derivative being of the same order in $n$ and uniform in $\alpha_{j}$ 's). Hence, we easily conclude that the error terms in (115), (126)-(128) have the same differentiability property. Alternatively, we could have deduced the differentiability of the error terms by noticing first that the asymptotic expansions of Bessel functions we used are differentiable in $\alpha_{j}$ 's.

\section{$5 \quad$ Proof of Theorem 1}

We now substitute the asymptotics (126) $-(128)$, and (115) into the differential identity (34). We assume first that all $\left\{\alpha_{j}\right\}_{j=1}^{m} \in \widetilde{\Omega} \backslash \hat{\Omega}$ (see Section 2) and $\{0\} \in \widetilde{\Omega}$. Care is needed with estimation of $\varkappa_{n}$. To obtain the asymptotics of $\varkappa_{n}$ from (126) we need first to replace $n$ with $n+1$ and second, to replace $\lambda_{j}$ with $\lambda_{j} \sqrt{\frac{n}{n+1}}$. Without this second step we would obtain a coefficient corresponding to the weight $\prod_{j}\left|x-\lambda_{j} \sqrt{2(n+1)}\right|^{2 \alpha_{j}} e^{-x^{2}}$. However, since the new $\lambda_{j}$ is inside $(-1,1)$ for $n$ large enough, and because it enters the asymptotics of $\varkappa_{n}$ starting 
with $O(1 / n)$ term, this second replacement affects only $t_{j}$ in (126). We obtain

$$
\begin{aligned}
& -\left(n+2 \sum_{j=1}^{m} \alpha_{j}\right)\left(\ln \varkappa_{n} \varkappa_{n-1}\right)_{\alpha_{\nu}}^{\prime}-2\left(\frac{\varkappa_{n-1}}{\varkappa_{n}}\right)^{2}\left(\ln \frac{\varkappa_{n-1}}{\varkappa_{n}}\right)_{\alpha_{\nu}}^{\prime}+ \\
& 2\left[\gamma_{n, \alpha_{\nu}}^{\prime}-\beta_{n} \beta_{n, \alpha_{\nu}}^{\prime}\right]=\left(n+2 \sum_{j=1}^{m} \alpha_{j}\right) \ln (n / 2)+\left(2 \lambda_{\nu}^{2}-1\right) n+2 \alpha_{\nu}+O(1 / n) .
\end{aligned}
$$

Furthermore,

$$
\begin{aligned}
& 2 \sum_{j=1}^{m} \alpha_{j}\left(Y_{11}\left(\mu_{j}\right)_{\alpha_{\nu}}^{\prime} Y_{22}^{\mathrm{vp}}\left(\mu_{j}\right)-Y_{21}\left(\mu_{j}\right)_{\alpha_{\nu}}^{\prime} Y_{12}^{\mathrm{vp}}\left(\mu_{j}\right)+\left(\ln \varkappa_{n} \varkappa_{n-1}\right)_{\alpha_{\nu}}^{\prime} Y_{11}\left(\mu_{j}\right) Y_{22}^{\mathrm{vp}}\left(\mu_{j}\right)\right)= \\
& \alpha_{\nu} \ln \left(1-\lambda_{\nu}^{2}\right)-2 \alpha_{\nu} \frac{\Gamma^{\prime}\left(\alpha_{\nu}+1 / 2\right)}{\Gamma\left(\alpha_{\nu}+1 / 2\right)}-2 \sum_{j \neq \nu} \alpha_{j} \ln \left(n\left|\lambda_{j}-\lambda_{\nu}\right|\right)+O\left(\frac{\ln n}{n}\right) .
\end{aligned}
$$

Note that the trigonometric terms of (126)-(128), and (115) cancel in these expressions.

Legitimacy of differentiation of the error terms w.r.t. $\alpha$ (and uniformity of these terms and their derivatives) follows from that for the error terms in (126) $-(128)$, and (115) explained in the end of the previous section.

The sum of (129) and (130) yields:

$$
\begin{aligned}
& \frac{d}{d \alpha_{\nu}} \ln D_{n}\left(\alpha_{1}, \alpha_{2}, \ldots, \alpha_{m}\right)=\left(n+2 \alpha_{\nu}\right) \ln (n / 2)+\left(2 \lambda_{\nu}^{2}-1\right) n+2 \alpha_{\nu}+\alpha_{\nu} \ln \left(1-\lambda_{\nu}^{2}\right)- \\
& 2 \alpha_{\nu} \frac{\Gamma^{\prime}\left(\alpha_{\nu}+1 / 2\right)}{\Gamma\left(\alpha_{\nu}+1 / 2\right)}-2 \sum_{j \neq \nu} \alpha_{j} \ln \left(2\left|\lambda_{j}-\lambda_{\nu}\right|\right)+O\left(\frac{\ln n}{n}\right), \quad \nu=1,2, \ldots, m .
\end{aligned}
$$

Since the error term here is uniform in all $\alpha_{\nu}$, we can integrate this identity.

First, set $\alpha_{2}=\cdots=\alpha_{m}=0, \nu=1$ in (131). This identity was established for $\alpha_{1}$ outside the set $\Omega(*, 0, \ldots, 0)$ (see Section 2 ). Note that the number of points in $\Omega(*, 0, \ldots, 0)$ is at most finite. Indeed, the function $\varkappa_{k}^{2}=\varkappa_{k}^{2}\left(\alpha_{1}, 0, \ldots, 0\right)$ is a ratio $D_{k} / D_{k+1}$ of two analytic functions of $\alpha_{1}$ for $\Re \alpha_{1}>-1 / 2$. They are not identically zero because they are known to be positive for $\alpha_{1}=0$. Consider the function $f\left(\alpha_{1}\right)=D_{n}\left(\alpha_{1}, 0, \ldots, 0\right) \exp \left(-\int_{0}^{\alpha_{1}} r(n, z) d z\right)$, where $r\left(n, \alpha_{1}\right)$ is the r.h.s. of (131) with $\nu=1, \alpha_{2}=\cdots=\alpha_{m}=0$. Equation (131) is the statement that $f^{\prime}\left(\alpha_{1}\right)=0$ outside $\Omega(*, 0, \ldots, 0)$. Since $f\left(\alpha_{1}\right)$ is continuously differentiable, $f^{\prime}\left(\alpha_{1}\right)=0$ for all $\alpha_{1}$ such that $\left(\alpha_{1}, 0, \ldots, 0\right) \in \widetilde{\Omega}$. Moreover, as $f\left(\alpha_{1}\right)=f(0)=$ $D_{n}(0, \ldots, 0) \neq 0$, the determinant $D_{n}\left(\alpha_{1}, 0, \ldots, 0\right)$ is never zero. Hence the identity (131) with $\nu=1, \alpha_{2}=\cdots=\alpha_{m}=0$ holds for all $\alpha_{1}$ such that $\left(\alpha_{1}, 0, \ldots, 0\right) \in \widetilde{\Omega}$. Integrating it over $\alpha_{1}$ from 0 to some $\alpha_{1}$, we obtain:

$$
\begin{aligned}
& \ln D_{n}\left(\alpha_{1}, 0, \ldots, 0\right)=\ln C\left(\alpha_{1}\right)+\frac{\alpha_{1}^{2}}{2} \ln \left(1-\lambda_{1}^{2}\right)+\left(\alpha_{1} n+\alpha_{1}^{2}\right) \ln \frac{n}{2}+n\left(2 \lambda_{1}^{2}-1\right) \alpha_{1}+ \\
& \ln D_{n}(0, \ldots, 0)+O\left(\frac{\ln n}{n}\right),
\end{aligned}
$$


where

$$
C(\alpha)=\Gamma(\alpha+1 / 2)^{-2 \alpha} \exp \left(2 \int_{0}^{\alpha} \ln \Gamma(s+1 / 2) d s+\alpha^{2}\right) .
$$

By freedom in the choice of $\widetilde{\Omega}$, the expansion (132) is valid for any $\alpha_{1}, \Re \alpha_{1}>-1 / 2$.

Second, a reasoning similar to the one above shows that equation (131) with $\alpha_{1}$ fixed and $\alpha_{3}=\cdots=\alpha_{m}=0$ holds for all $\alpha_{2}, \Re \alpha_{2}>-1 / 2$. Integrating it over $\alpha_{2}$ from 0 to some $\alpha_{2}$ gives

$$
\begin{aligned}
& \ln D_{n}\left(\alpha_{1}, \alpha_{2}, 0, \ldots, 0\right)=\ln C\left(\alpha_{2}\right)+\frac{\alpha_{2}^{2}}{2} \ln \left(1-\lambda_{2}^{2}\right)+\left(\alpha_{2} n+\alpha_{2}^{2}\right) \ln \frac{n}{2}+n\left(2 \lambda_{2}^{2}-1\right) \alpha_{2} \\
& -2 \alpha_{1} \alpha_{2} \ln 2\left|\lambda_{1}-\lambda_{2}\right|+\ln D_{n}\left(\alpha_{1}, 0, \ldots, 0\right)+O\left(\frac{\ln n}{n}\right)= \\
& \ln C\left(\alpha_{1}\right) C\left(\alpha_{2}\right)+\sum_{j=1}^{2} \frac{\alpha_{j}^{2}}{2} \ln \left(1-\lambda_{j}^{2}\right)+\sum_{j=1}^{2}\left(\alpha_{j} n+\alpha_{j}^{2}\right) \ln \frac{n}{2}+n \sum_{j=1}^{2} \alpha_{j}\left(2 \lambda_{j}^{2}-1\right) \\
& -2 \alpha_{1} \alpha_{2} \ln 2\left|\lambda_{1}-\lambda_{2}\right|+\ln D_{n}(0, \ldots, 0)+O\left(\frac{\ln n}{n}\right) .
\end{aligned}
$$

To obtain the second equation, we substituted $\ln D_{n}\left(\alpha_{1}, 0, \ldots, 0\right)$ from (132). Equation (134) is valid for any $\alpha_{1}, \alpha_{2}, \Re \alpha_{1}, \Re \alpha_{2}>-1 / 2$.

Proceeding in this way, we prove Theorem 1 by induction after $m$ steps.

The second representation (6) for the constant $C(\alpha)$ is easy to obtain using the following properties of the G-function [35]:

$$
\begin{aligned}
& G(z+1)=\Gamma(z) G(z), \quad G(1)=1 \\
& \int_{0}^{z} \ln \Gamma(x+1) d x=\frac{z}{2} \ln 2 \pi-\frac{z(z+1)}{2}+z \ln \Gamma(z+1)-\ln G(z+1) .
\end{aligned}
$$

Another identity is useful for comparison with (4):

$$
2 \ln G(1 / 2)=(1 / 12) \ln 2-\ln \sqrt{\pi}+3 \zeta^{\prime}(-1) .
$$

\section{Acknowledgements}

I thank Y. Fyodorov (from whom I learned about this problem) and A. Kuijlaars for useful discussions and comments. I am very grateful to P. Deift and A. Its for many important suggestions, and to V. Kravtsov and O. Yevtushenko for their hospitality at the Abdus Salam ICTP where this work was started. 


\section{References}

[1] Handbook of Mathematical Functions (ed. M. Abramowitz, I. Stegun), NY: Dover, 1965

[2] J. Baik, P. Deift, K. Johansson: On the Distribution of the Length of the Longest Increasing Subsequence of Random Permutations, J. Amer. Math. Soc. 12, 1119-1178 (1999)

[3] J. Baik, P. Deift, E. Strahov: Products and ratios of characteristic polynomials of random Hermitian matrices. J. Math. Phys. 44 3657-3670 (2003)

[4] P. M. Bleher, A. B. J. Kuijlaars: Random matrices with external source and multiple orthogonal polynomials. Int. Math. Res. Notices 2004 (3), 109-129 (2004) math-ph/0307055

[5] Pavel M. Bleher, Alexander R. Its: Asymptotics of the partition function of a random matrix model. Ann. Inst. Fourier (Grenoble) 55, no. 6, 1943-2000 (2005)

[6] A. Borodin, E. Strahov: Averages of characteristic polynomials in random matrix theory. Comm. Pure Appl. Math. 59 161-253 (2006)

[7] E. Brézin, S. Hikami: Characteristic polynomials of random matrices. Commun. Math. Phys. 214, 111-135 (2000)

[8] P. Deift: Orthogonal polynomials and random matrices: a Riemann-Hilbert approach. Courant Lecture Notes in Math. 1998

[9] P. Deift: Talk at the conference "Spectral theory and inverse spectral theory for Jacobi operators", Snowbird, June 2003.

[10] P. Deift and X. Zhou: A steepest descent method for oscillatory Riemann-Hilbert problem. Ann. Math. 137, 295-368 (1993)

[11] P. Deift, A. Its, and X. Zhou: A Riemann-Hilbert approach to asymptotic problems arising in the theory of random matrix models, and also in the theory of integrable statistical mechanics. Ann. Math 146, 149-235 (1997)

[12] P. Deift, T. Kriecherbauer, K. T-R McLaughlin, S. Venakides, X. Zhou: Strong asymptotics for orthogonal polynomials with respect to exponential weights. Commun. Pure Appl. Math. 52, 1491-1552 (1999)

[13] P. Deift, A. Its, I. Krasovsky, X. Zhou: The Widom-Dyson constant and related questions of the asymptotic analysis of Toeplitz determinants. J. Comput. Appl. Math. 202, 26-47 (2007) math.FA/0601535

[14] P. Deift, A. Its, I. Krasovsky: Asymptotics of the Airy-kernel determinant. math.FA/0609451 
[15] N. M. Ercolani, K. D. T.-R. McLaughlin: Asymptotics of the partition function for random matrices via Riemann-Hilbert techniques and applications to graphical enumeration. Int. Math. Res. Not. 2003, 755-820 (2003).

[16] A. S. Fokas, A. R. Its, A. V. Kitaev: The isomonodromy approach to matrix models in 2D quantum gravity. Commun. Math. Phys. 147, 395-430 (1992)

[17] P. J. Forrester, N. E. Frankel: Applications and generalizations of Fisher-Hartwig asymptotics. J. Math. Phys. 45 (No.5), 2003-20028 (2004) [arXiv: math-ph/0401011].

[18] P. J. Forrester, N. E. Frankel, T.M. Garoni, N.S. Witte: Finite one dimensional impenetrable Bose systems: Occupation numbers. Phys. Rev A 67 (4), 043607 (2003) [arXiv: cond-mat/0211126

[19] Y. V. Fyodorov: Complexity of random energy landscapes, glass transition and the absolute value of spectral determinant of random matrices. Physical Review Letters 92 (No.24) article No. 240601 (2004); Erratum: ibid. 93 (No.14) article No. 149901(E) (2004) cond-mat/0401287

[20] D. M. Gangardt: Universal correlations of trapped one-dimensional impenetrable bosons. J.Phys.A: Math. Gen. 37 (No.40), 9335-9356 (2004) cond-mat/0404104

[21] T. M. Garoni: On the asymptotics of some large Hankel determinants generated by Fisher-Hartwig symbols defined on the real line. J. Math. Phys. 46 043516, 19 pp. (2005)

[22] K. Johansson: On fluctuations of eigenvalues of random hermitian matrices. Duke Math. J. 91, 151-204 (1998)

[23] J. P. Keating, N. C. Snaith: Random matrix theory and $\zeta(1 / 2+i t)$. Commun. Math. Phys. 214, 57-89 (2000)

[24] J.P. Keating, N. Linden, Z. Rudnick: Random matrix theory, the exceptional Lie groups and $L$-functions. J. Phys. A 36, 2933-2944 (2003)

[25] I. V. Krasovsky: Gap probability in the spectrum of random matrices and asymptotics of polynomials orthogonal on an arc of the unit circle. Int. Math. Res. Not. 2004, 1249-1272 (2004) [math.FA/0401258

[26] T. Kriecherbauer, K. T-R McLaughlin: Strong asymptotics of polynomials orthogonal with respect to Freud weights, Int. Math. Res. Not. 1999, 299-333 (1999)

[27] A. B. J. Kuijlaars, M. Vanlessen: Universality for eigenvalue correlations at the origin of the spectrum. Commun. Math. Phys. 243, 163-191 (2003) 
[28] A. B. J. Kuijlaars, K. T-R McLaughlin, W. Van Assche, M. Vanlessen: The RiemannHilbert approach to strong asymptotics for orthogonal polynomials on $[-1,1]$. Adv. Math. 188, 337-398 (2004) [math.CA/011125]

[29] M. L. Mehta: Random matrices. San Diego: Academic 1990

[30] M. L. Mehta, J. -M. Normand: Moments of the characteristic polynomial in the three ensembles of random matrices. J.Phys. A 34 4627-4639 (2001)

[31] E. Strahov, Y. V. Fyodorov: Universal results for correlations of characteristic polynomials: Riemann-Hilbert approach. Commun.Math.Phys. 241 (No.2-3), 343-382 (2003) math-ph/0210010

[32] G. Szegő: Orthogonal polynomials. AMS Colloquium Publ. 23. New York: AMS 1959

[33] M. Vanlessen: Universal behavior for averages of characteristic polynomials at the origin of the spectrum. Commun. Math. Phys. 253, 535-560 (2005) math-ph/0306078

[34] M. Vanlessen: Strong asymptotics of the recurrence coefficients of orthogonal polynomials associated to the generalized Jacobi weight. J. Approx. Theory 125, 198-237 (2003)

[35] E. Whittaker, G. Watson: A course of modern analysis, Cambridge, 1969.

[36] H. Widom: The strong Szegö limit theorem for circular arcs. Indiana Univ. Math. J. 21, 277-283 (1971)

[37] H. Widom: Toeplitz determinants with singular generating functions. Amer. J. Math. $95333-383$ (1973) 